\title{
Lie-Hamilton systems on curved spaces: A geometrical approach
}

\author{
Francisco J. Herranz ${ }^{1}$, Javier de Lucas $^{2}$ And Mariusz Tobolski ${ }^{3}$ \\ ${ }^{1}$ Department of Physics, University of Burgos, 09001, Burgos, Spain \\ ${ }^{2}$ Department of Mathematical Methods in Physics, University of Warsaw, Pasteura 5, 02-093, Warszawa, \\ Poland \\ ${ }^{3}$ Institute of Mathematics, Polish Academy of Sciences, ul. Śniadeckich 8, 00-656, Warszawa, Poland \\ E-mail: fjherranz@ubu.es, javier.de.lucas@fuw.edu.pl,mtobolski@impan.pl
}

\begin{abstract}
A Lie-Hamilton system is a nonautonomous system of first-order ordinary differential equations describing the integral curves of a $t$-dependent vector field taking values in a finite-dimensional Lie algebra, a Vessiot-Guldberg Lie algebra, of Hamiltonian vector fields relative to a Poisson structure. Its general solution can be written as an autonomous function, the superposition rule, of a generic finite family of particular solutions and a set of constants. We pioneer the study of Lie-Hamilton systems on Riemannian spaces (sphere, Euclidean and hyperbolic plane), pseudo-Riemannian spaces (anti-de Sitter, de Sitter, and Minkowski spacetimes) as well as on semi-Riemannian spaces (Newtonian spacetimes). Their corresponding constants of motion and superposition rules are obtained explicitly in a geometric way. This work extends the (graded) contraction of Lie algebras to a contraction procedure for Lie algebras of vector fields, Hamiltonian functions, and related symplectic structures, invariants, and superposition rules.
\end{abstract}

KEYWORDS: Cayley-Klein geometries, graded contraction, Lie system, Poisson coalgebra, pseudoRiemannian space, superposition rule, symplectic geometry

PACS: 02.20.Sv, 02.40.Dr, 02.40.Ky

MSC2010: 34A26 (primary), 17B66, 70G45, 34A34 (secondary)

\section{Introduction}

A Lie system is a nonautonomous system of first-order ordinary differential equations describing the integral curves of a $t$-dependent vector field taking values in a finite-dimensional Lie algebra of vector fields, a so-called Vessiot-Guldberg Lie algebra [1, 2]. The Lie-Scheffers theorem [3] establishes that a Lie system amounts to a nonautonomous system of first-order ordinary differential equations whose general solution can be written as an autonomous function, a superposition rule, of a generic family of particular solutions and some constants related to initial conditions [1, 2, 3, 4, 5, 6]. 
Some relevant examples of Lie systems are nonautonomous systems of first-order linear ordinary differential equations [2] and matrix Riccati equations [4]. Although most differential equations cannot be described through Lie systems [2, 7, Lie systems occur in relevant physical and mathematical problems, such as Winternitz-Smorodinsky oscillators and Ermakov systems [2], which motivates their study (see 2, 8, 9, for more applications).

Lie systems admitting a Vessiot-Guldberg Lie algebra of Hamiltonian vector fields relative to a Poisson structure [10, 11] are called Lie-Hamilton (LH) systems [12. Although Lie systems and LH systems have been widely studied and applied, most of them are defined on a flat Euclidean space $\mathbb{R}^{n}$ [8, 9, 13, 14, 15. In particular, their local classification on $\mathbb{R}^{2}$ has been recently established in 8 , starting from the classification of finite-dimensional Lie algebras of vector fields on $\mathbb{R}^{2}$ up to a local diffeomorphism developed by González, Kamran, and Olver, the so-called GKO classification 16. Constants of motion and superposition rules for LH systems on $\mathbb{R}^{2}$ were studied in 9 .

There exists an almost complete classification and derivation of superposition rules for complex Lie systems with primitive transitive Vessiot-Guldberg Lie algebras of vector fields on homogeneous spaces due to Winternitz and collaborators [17, 18. In spite of that, there are not many results for Lie systems possessing general real Vessiot-Guldberg Lie algebras on homogeneous spaces, which represents a much more complicated problem (cf. [17, 18). Some results can be found on one- and two-dimensional spheres [1, 19, 20, 21]. Relevantly, the so-called $t$-dependent projective Schrödinger equations are Lie systems on a complex projective space admitting a real Vessiot-Guldberg Lie algebra of Lie symmetries of a Riemannian metric with positive constant curvature. This Vessiot-Guldberg Lie algebra also consists of Hamiltonian vector fields relative to a symplectic structure coming from the quantum mechanical structure of the problem 22. Due to the lack of research on Lie systems in real manifolds, this paper aims to fill this gap in the literature by classifying LH systems on two-dimensional (2D) spaces with a Vessiot-Guldberg Lie algebra of Lie symmetries of a (possibly degenerate) metric of constant curvature by following a geometrical approach which also enables one to calculate their constants of motion and superposition rules explicitly.

Section 2 surveys Lie systems on $\mathbb{R}^{2}$ possessing a Vessiot-Guldberg Lie algebra $V$ belonging to the class $\mathrm{P}_{1}$ of the GKO classification [8, 9]. Such a Vessiot-Guldberg Lie algebra is isomorphic to the 2D Euclidean algebra iso(2). The vector fields of $V$ become Hamiltonian relative to a symplectic structure on $\mathbb{R}^{2}$. Moreover, $V$ consists of Lie symmetries of the metric of the Euclidean plane $\mathbf{E}^{2}:=\mathbb{R}^{2}$. This allows us to obtain the corresponding superposition rules by using Euclidean and symplectic geometry [9].

To generalize the latter results to other (curved) spaces, we give in section 3 a brief account on the nine 2D Cayley-Klein $(C K)$ spaces $\left[23,24,25,26,27\right.$, which are collectively denoted by $\mathbf{S}_{\left[\kappa_{1}\right], \kappa_{2}}^{2}$ where $\kappa_{1}$ and $\kappa_{2}$ are two real parameters. The former is just the constant Gaussian curvature of the space, while the latter determines the metric of the space through $\operatorname{diag}\left(+1, \kappa_{2}\right)$. Therefore, the CK spaces cover the three classical Riemannian spaces of constant curvature for $\kappa_{2}>0$ (sphere, Euclidean, and hyperbolic spaces), three pseudo-Riemannian or Lorentzian spaces for $\kappa_{2}<0$ (anti-de Sitter, Minkowski, and de Sitter spaces), as well as three semi-Riemannian or Newtonian spaces, so with a degenerate metric, for $\kappa_{2}=0$. The Euclidean plane $\mathbf{E}^{2}$ corresponds to the CK space $\mathbf{S}_{[0],+}^{2}$.

In section 4 , new Lie systems on $\mathbf{S}_{\left[\kappa_{1}\right], \kappa_{2}}^{2}$ are obtained by considering the Lie algebras of Lie symmetries of the metric on $\mathbf{S}_{\left[\kappa_{1}\right], \kappa_{2}}^{2}$ in the so-called geodesic parallel coordinates [28]; these are a natural generalization of the Cartesian coordinates to curved spaces. Next a symplectic form is found to turn previous Lie symmetries into Hamiltonian vector fields, so providing LH systems on $\mathbf{S}_{\left[\kappa_{1}\right], \kappa_{2}}^{2}$.

Previous new Lie systems admit Vessiot-Guldberg Lie algebras of conformal vector fields on twodimensional manifolds. Although superposition rules for Lie systems on linear spaces admitting VessiotGuldberg Lie algebras of conformal vector fields can be found in [9, 14, 15], such results cannot effectively be applied to the Lie systems here proposed for a number of reasons. First, there exists no global diffeomorphism mapping Lie systems on manifolds to particular cases of the Lie systems treated in [9, 14, 15] because, for instance, there is no diffeomorphism from a sphere to a linear space. As a consequence, there is no way to apply the superposition rules derived in [9, 14, 15, to our Lie systems. Second, the Lie systems studied in [14, 15] are related to Vessiot-Guldberg Lie algebras of larger dimension than those given in the present work. This causes the related superposition rules to depend on a larger number of particular solutions and to have different properties than ours 2]. Finally, our approach is 
specially adapted to the geometry of the manifold where the Lie systems are defined. This involves the use of special spherical trigonometric functions and other techniques. This cannot be achieved through methods in [9, 14, 15], as they do not consider the geometry of $\mathbf{S}_{\left[\kappa_{1}\right], \kappa_{2}}^{2}$.

Previous results are completed in section 5 , where, firstly, $t$-independent constants of motion are obtained by applying the Poisson coalgebra approach introduced in [13] and, secondly, superposition rules are deduced by making use of trigonometry on such (curved) spaces [29].

Lie algebras of vector fields, Hamiltonian functions, and related structures appearing in sections 3, 4, and 5 are parametrized in terms of the parameters $\kappa_{a}(a=1,2)$. Such expressions are illustrated for each specific space in tables 1 and 2, which summarize the main results of the paper. The cases with $\kappa_{1}=0$ and $\kappa_{2}>0$ recover known results on Euclidean LH systems on the plane [8]. Moreover, this allows us to generalize graded contractions of abstract Lie algebras [30, 31, 32], which comprise the Inönü-Wigner Lie algebra contractions corresponding to the limits $\kappa_{a} \rightarrow 0$, to Lie algebras of vector fields, Hamiltonian functions, etc. This highlights transitions among all of these known and new LH systems and their associated structures. In fact, contractions of Lie systems have only been considered very recently in [33, but a systematic use covering contractions of vector fields, symplectic structures, constants of motion, and superposition rules was still lacking. Finally, some open problems close the paper.

\section{A class of Lie-Hamilton systems on the Euclidean plane}

Let us consider the Euclidean plane $\mathbf{E}^{2}:=\mathbb{R}^{2}$ with global coordinates $\{x, y\}$ along with a real parameter $t$ and a nonautonomous system of first-order differential equations

$$
\frac{\mathrm{d} x}{\mathrm{~d} t}=f(t, x, y), \quad \frac{\mathrm{d} y}{\mathrm{~d} t}=g(t, x, y),
$$

where $f, g: \mathbb{R}^{3} \rightarrow \mathbb{R}$ are arbitrary functions. System (2.1) is geometrically described by the $t$-dependent vector field

$$
X:(t, x, y) \in \mathbb{R} \times \mathbb{R}^{2} \mapsto f(t, x, y) \frac{\partial}{\partial x}+g(t, x, y) \frac{\partial}{\partial y} \in \mathrm{TR}^{2} .
$$

Conversely, the above $t$-dependent vector field induces a unique nonautonomous system of differential equations determining its integral curves given by (2.1) (see [2]). This justifies the use of $X$ to refer to both (2.1) and (2.2). A Lie system on $\mathbb{R}^{2}$ is a system of the form

$$
X_{t}(x, y):=X(t, x, y)=\sum_{i=1}^{l} b_{i}(t) X_{i}(x, y),
$$

where $b_{1}(t), \ldots, b_{l}(t)$ are some $t$-dependent real functions and $X_{1}, \ldots, X_{l}$ are vector fields on $\mathbb{R}^{2}$ spanning an $l$-dimensional real Lie algebra $V$, a Vessiot-Guldberg Lie algebra of $X$. The Lie-Scheffers Theorem [3, 4, 5] states that a nonautonomous system of first-order ordinary differential equations is a Lie system if and only if its general solution can be described through a superposition rule [1, 2, 3, 4,

For our purposes and to illustrate the above concepts, we consider the $t$-dependent vector field on $\mathbb{R}^{2}$ of the form

$$
X:=b_{1}(t) X_{1}+b_{2}(t) X_{2}+b_{3}(t) X_{3}
$$

where $b_{1}(t), b_{2}(t), b_{3}(t)$ are arbitrary $t$-dependent functions and

$$
X_{1}:=\frac{\partial}{\partial x}, \quad X_{2}:=\frac{\partial}{\partial y}, \quad X_{3}:=y \frac{\partial}{\partial x}-x \frac{\partial}{\partial y} .
$$

Hence, $X$ is related to a system of nonautonomous first-order ordinary differential equations

$$
\frac{\mathrm{d} x}{\mathrm{~d} t}=b_{1}(t)+b_{3}(t) y, \quad \frac{\mathrm{d} y}{\mathrm{~d} t}=b_{2}(t)-b_{3}(t) x .
$$

The previous system can be rewritten as a linear inhomogeneous complex differential equation

$$
\frac{\mathrm{d} z}{\mathrm{~d} t}=\left(b_{1}(t)+\mathrm{i} b_{2}(t)\right)-\mathrm{i} b_{3}(t) z
$$


admitting a complex Vessiot-Guldberg Lie algebra $V_{C}=\left\langle\partial_{z}, z \partial_{z}\right\rangle_{\mathbb{C}}$ isomorphic to the complex affine Lie algebra $\mathfrak{A f f}(\mathbb{C})$. Meanwhile, complex Bernoulli equations [34] of order $\alpha \in \mathbb{R} \backslash\{1\}$, namely

$$
\frac{\mathrm{d} w}{\mathrm{~d} t}=a(t) w+b(t) w^{\alpha}
$$

for arbitrary complex functions $a(t)$ and $b(t)$, possess a complex Vessiot-Guldberg Lie algebra $V_{C B}=$ $\left\langle w \partial_{w}, w^{\alpha} \partial_{w}\right\rangle \simeq \mathfrak{A f f}(\mathbb{C})$. On the one hand, since affine Lie algebras of complex vector fields on the complex line are diffeomorphic, there exists a complex change of variables mapping one onto the other, namely $w^{1-\alpha}=z$. This maps (2.5), written as a complex inhomogeneous differential equation, onto a complex Bernoulli equation. Moreover, complex Bernoulli equations, as real Lie systems, admit a Vessiot-Guldberg Lie algebra given by the realification of $V_{C B}$, denoted by $V_{C B}^{R}$, which retrieves a result in [9. In consequence, (2.5) must admit a Vessiot-Guldberg Lie algebra isomorphic to a Lie subalgebra of $V_{C B}^{R}$.

The vector fields (2.4) span a 3D real Vessiot-Guldberg Lie algebra $V$ with commutation relations

$$
\left[X_{3}, X_{1}\right]=X_{2}, \quad\left[X_{3}, X_{2}\right]=-X_{1}, \quad\left[X_{1}, X_{2}\right]=0 .
$$

That is, $V$ can be written as a semidirect sum $V \simeq \mathfrak{s o}(2) \ltimes \mathbb{R}^{2} \simeq\left\langle X_{3}\right\rangle \ltimes\left\langle X_{1}, X_{2}\right\rangle$, so being isomorphic to the 2D Euclidean Lie algebra iso(2). Consequently, $X$ is called an iso(2)-Lie system.

The Lie algebra $V$ belongs to the class $\mathrm{P}_{1}$ of the GKO classification [8, 16. The system $X$ is therefore called a $P_{1}$-Lie system. Additionally, $\mathrm{P}_{1}$ is also one of the 12 classes of finite-dimensional real Lie algebras of Hamiltonian vector fields on $\mathbb{R}^{2}$ according to the classification performed in [8, 9]. Hence, $X$ admits a Vessiot-Guldberg Lie algebra of Hamiltonian vector fields with respect to a Poisson structure [10, 11, and it becomes an iso(2)-LH system. In particular, the vector fields of $V$ are Hamiltonian relative to the (canonical) symplectic form

$$
\omega=\mathrm{d} x \wedge \mathrm{d} y .
$$

Their corresponding Hamiltonian functions, $h_{i}$, can be obtained by using the relation $\iota_{X_{i}} \omega=\mathrm{d} h_{i}(i=$ $1,2,3)$; these can be chosen to be [8, 9]

$$
h_{1}:=y, \quad h_{2}:=-x, \quad h_{3}:=\frac{1}{2}\left(x^{2}+y^{2}\right) .
$$

Thus,

$$
h_{t}:=b_{1}(t) h_{1}+b_{2}(t) h_{2}+b_{3}(t) h_{3}
$$

is a Hamiltonian function associated with the vector field $X_{t}$ given by (2.3) for every $t \in \mathbb{R}$. The linear space $\left\langle h_{1}, h_{2}, h_{3}\right\rangle$ can be expanded to a finite-dimensional Lie algebra of functions, relative to the Poisson bracket $\{\cdot, \cdot\}_{\omega}$ related to $\omega$, by adding a new Hamiltonian function $h_{0}:=1$. In this way,

$$
\left\{h_{3}, h_{1}\right\}_{\omega}=-h_{2}, \quad\left\{h_{3}, h_{2}\right\}_{\omega}=h_{1}, \quad\left\{h_{1}, h_{2}\right\}_{\omega}=h_{0}, \quad\left\{h_{0}, \cdot\right\}_{\omega}=0,
$$

and $\left\langle h_{1}, h_{2}, h_{3}, h_{0}\right\rangle$ becomes a Lie algebra (with respect to $\{\cdot, \cdot\}_{\omega}$ ) isomorphic to the centrally extended Euclidean Lie algebra $\overline{\mathfrak{i s o}}(2)$. This term is coined due to the fact that there exists an exact Lie algebra sequence

$$
\left\langle h_{0}\right\rangle \hookrightarrow\left\langle h_{1}, h_{2}, h_{3}, h_{0}\right\rangle \stackrel{\phi}{\rightarrow} V \simeq \mathfrak{i s o}(2)
$$

where $\phi\left(h_{i}\right)=X_{i}$ and $\phi\left(h_{0}\right)=0$. Remarkably, every Lie algebra containing the Hamiltonian functions for the vector fields of $V$ will generate a Lie algebra isomorphic to $\overline{\mathfrak{i s o}}(2)$ (cf. [8, Corollary 5.4 and Proposition 5.5]). We call this Lie algebra a $L H$ algebra, $\mathcal{H}_{\omega}$, for the LH system $X$.

\subsection{Constants of motion and superposition rules}

When a nonautonomous system of first-order ordinary differential equations $X$ is shown to be a Lie system, the Lie-Scheffers Theorem ensures that it possesses a superposition rule which can be deduced by standard (but generally cumbersome) methods [2, 4, 5. If $X$ is a LH system, there also exists 
an alternative Poisson coalgebra approach, which enables one to obtain the corresponding constants of motion (invariants) and superposition rules in an easier geometric manner. This procedure has recently been formulated in [13] and extensively applied in [9] to the 12 classes of LH systems on the Euclidean plane. In what follows, we review the essentials of such a Poisson coalgebra procedure by applying it to the $\mathrm{P}_{1}$-Lie system $X$ given by (2.4) (see 9 , 13 , for details).

Let $S(\overline{\mathfrak{i s o}}(2))$ be the symmetric algebra of $\overline{\mathfrak{i s o}}(2)$ [35, 36, i.e. the algebra of polynomial functions on the elements of $\overline{\mathfrak{i s o}}(2)$. Let $\left\{v_{0}, v_{1}, v_{2}, v_{3}\right\}$ be a basis of $\overline{\mathfrak{i s o}}(2)$ fulfilling the commutation relations (2.8). The Lie bracket on $\overline{\mathfrak{i s o}}(2) \subset S(\overline{\mathfrak{i s o}}(2))$ can be extended in a unique way to a Poisson bracket $\{\cdot, \cdot\}_{S}$ on $S(\overline{\mathfrak{i s o}}(2))$, which becomes a Poisson algebra [10, 13]. Then, $S(\overline{\mathfrak{i s o}}(2))$ has a second-order Casimir invariant 37 .

$$
C:=v_{3} v_{0}-\frac{1}{2}\left(v_{1}^{2}+v_{2}^{2}\right),
$$

i.e. $C$ is a quadratic function in the variables $v_{0}, v_{1}, v_{2}, v_{3}$, and $\{C, w\}_{S}=0$ for every $w \in S(\overline{\mathbf{i s o}}(2))$. The tensor product of Poisson algebras becomes a Poisson algebra in a canonic way [10], and $S(\overline{\mathfrak{i n o}}(2))$ can be endowed with a Poisson coalgebra [13] structure by means of the non-deformed coproduct map $\Delta: S(\overline{\mathfrak{i s o}}(2)) \rightarrow S(\overline{\mathfrak{i s o}}(2)) \otimes S(\overline{\mathfrak{i s o}}(2))$ defined by requiring $\Delta$ to be a linear morphism such that $\Delta(w v)=\Delta(w) \Delta(v)$ for every $w, v \in S(\overline{\mathfrak{i s o}}(2))$ and

$$
\Delta\left(v_{a}\right):=v_{a} \otimes 1+1 \otimes v_{a}, \quad a=0,1,2,3,
$$

namely $S(\overline{\mathfrak{i s o}}(2))$ is a Poisson algebra and $\Delta$ is a Poisson algebra homomorphism. The Poisson algebra morphisms $D: S(\overline{\mathfrak{i s o}}(2)) \rightarrow C^{\infty}\left(\mathbb{R}^{2}\right)$ and $D^{(2)}: S(\overline{\mathfrak{i s o}}(2)) \otimes S(\overline{\mathfrak{i s o}}(2)) \rightarrow C^{\infty}\left(\mathbb{R}^{2}\right) \otimes C^{\infty}\left(\mathbb{R}^{2}\right)$ defined by

$$
D\left(v_{a}\right):=h_{a}\left(x_{1}, y_{1}\right), \quad D^{(2)}\left(v_{a} \otimes 1\right):=h_{a}\left(x_{1}, y_{1}\right), \quad D^{(2)}\left(1 \otimes v_{a}\right):=h_{a}\left(x_{2}, y_{2}\right),
$$

where $h_{a}$ are the Hamiltonian functions (2.7), lead to the following $t$-independent constants of motion $F^{(1)}:=F$ and $F^{(2)}$ for the system $X$ through the Casimir $C$ as follows (see [13, Theorem 26] for details)

$$
F:=D(C), \quad F^{(2)}:=D^{(2)}(\Delta(C)),
$$

namely 9 ]

$$
F=0, \quad F^{(2)}=\frac{1}{2}\left[\left(x_{1}-x_{2}\right)^{2}+\left(y_{1}-y_{2}\right)^{2}\right] .
$$

The previous functions become constant when evaluated on pairs $\left(x_{i}(t), y_{i}(t)\right)$, with $i=1,2$, of particular solutions to $X$. Hence, they are first-integrals of the so-called diagonal prolongation of the $t$-dependent vector field $X$ to $\left(\mathbb{R}^{2}\right)^{2}\left[2,5\right.$, namely the $t$-dependent vector field on $\left(\mathbb{R}^{2}\right)^{2}$ given by

$$
\tilde{X}\left(t, x_{1}, y_{1}, x_{2}, y_{2}\right):=\sum_{i=1}^{2} \sum_{\alpha=1}^{3} b_{\alpha}(t) X_{\alpha}\left(x_{i}, y_{i}\right) .
$$

By permuting $x_{1} \leftrightarrow x_{3}, y_{1} \leftrightarrow y_{3}$ and $x_{2} \leftrightarrow x_{3}, y_{2} \leftrightarrow y_{3}$ in $F^{(2)}$, we find two functions $F_{13}^{(2)}, F_{23}^{(2)}:\left(\mathbb{R}^{2}\right)^{3} \rightarrow$ $\mathbb{R}$ of the form

$$
\left\{\begin{array}{l}
F_{13}^{(2)}:=\frac{1}{2}\left[\left(x_{3}-x_{2}\right)^{2}+\left(y_{3}-y_{2}\right)^{2}\right], \\
F_{23}^{(2)}:=\frac{1}{2}\left[\left(x_{1}-x_{3}\right)^{2}+\left(y_{1}-y_{3}\right)^{2}\right] .
\end{array}\right.
$$

Since the diagonal prolongation of $X$ to $\left(\mathbb{R}^{2}\right)^{3}$ is invariant under the permutation of variables, $F_{13}^{(2)}$ and $F_{23}^{(2)}$ are its first-integrals.

Since $\partial\left(F^{(2)}, F_{23}^{(2)}\right) / \partial\left(x_{1}, y_{1}\right) \neq 0$, both constants of motion are functionally independent (the pair $F^{(2)}, F_{13}^{(2)}$ is so as well). This condition allows us to solve the system of equations

$$
F^{(2)}=\frac{1}{2} k_{1}^{2} \geq 0, \quad F_{23}^{(2)}=\frac{1}{2} k_{2}^{2} \geq 0, \quad F_{13}^{(2)}=\frac{1}{2} k_{3}^{2}>0,
$$

in the variables $x_{1}, y_{1}$. In turn, we can write a function $\Phi:\left(x_{2}, y_{2} ; x_{3}, y_{3} ; k_{1}, k_{2}\right) \in \mathbb{R}^{2} \times \mathbb{R}^{2} \times \overline{\mathbb{R}}_{0}^{2} \mapsto$ $\left(x_{1}, y_{1}\right) \in \mathbb{R}^{2}$, where $\overline{\mathbb{R}}_{0}:=\{x \in \mathbb{R}, x \geq 0\}$. The theory of Lie systems [2] ensures that $\Phi$ enables us to 
write the general solution, $\left(x_{1}(t), y_{1}(t)\right)$ to $X$ as a function of two particular solutions $\left(x_{i}(t), y_{i}(t)\right)$ to $X$ and two constants, $k_{1}, k_{2}$, to be related to initial conditions as follows

$$
\left(x_{1}(t), y_{1}(t)\right)=\Phi\left(x_{2}(t), y_{2}(t), x_{3}(t), y_{3}(t), k_{1}, k_{2}\right) .
$$

In particular, the system of equations (2.13) admits two solutions in the variables $x_{1}, y_{1}$ according to the signs ' \pm ' $[9]$

$$
\left\{\begin{array}{c}
x_{1}^{ \pm}\left(x_{2}, y_{2}, x_{3}, y_{3}, k_{1}, k_{2}\right)=x_{2}+\frac{k_{1}^{2}+k_{3}^{2}-k_{2}^{2}}{2 k_{3}^{2}}\left(x_{3}-x_{2}\right) \mp 2 A \frac{\left(y_{3}-y_{2}\right)}{k_{3}^{2}} \\
y_{1}^{ \pm}\left(x_{2}, y_{2}, x_{3}, y_{3}, k_{1}, k_{2}\right)=y_{2}+\frac{k_{1}^{2}+k_{3}^{2}-k_{2}^{2}}{2 k_{3}^{2}}\left(y_{3}-y_{2}\right) \pm 2 A \frac{\left(x_{3}-x_{2}\right)}{k_{3}^{2}} \\
A=\frac{1}{4} \sqrt{2\left(k_{1}^{2} k_{2}^{2}+k_{1}^{2} k_{3}^{2}+k_{2}^{2} k_{3}^{2}\right)-\left(k_{1}^{4}+k_{2}^{4}+k_{3}^{4}\right)}
\end{array}\right.
$$

where $k_{3}^{2}=\left(x_{3}-x_{2}\right)^{2}+\left(y_{3}-y_{2}\right)^{2}$. Then, it is guaranteed [5] that the above expressions give rise to superposition rules $\Phi_{ \pm}:\left(x_{2}, y_{2} ; x_{3}, y_{3} ; k_{1}, k_{2}\right) \in \mathbb{R}^{2} \times \mathbb{R}^{2} \times \overline{\mathbb{R}}_{0}^{2} \mapsto\left(x_{1}^{ \pm}, y_{1}^{ \pm}\right) \in \mathbb{R}^{2}$.

Remarkably, above results admit a geometrical interpretation. The vector fields (2.4) are the infinitesimal generators of the isometries on the Euclidean plane $\mathbf{E}^{2}$ relative to the standard metric $\mathrm{d} x^{2}+\mathrm{d} y^{2}$. In particular, $X_{3}:=J_{12}$ is the generator of rotations around the origin on $\mathbf{E}^{2}$ (or the angular momentum), meanwhile $X_{1}:=P_{1}$ and $X_{2}:=P_{2}$ behave as infinitesimal generators of translations along the two basic axes $x$ and $y$, respectively.

Likewise, the invariants (2.11), (2.12), and the superposition rules (2.14) can also be geometrically described 9 . Let $k_{1}, k_{2}$ and $k_{3}$ be the Euclidean lengths of the segments $\overline{Q_{1} Q_{2}}, \overline{Q_{1} Q_{3}}$, and $\overline{Q_{2} Q_{3}}$ between the three points $Q_{1}:=\left(x_{1}, y_{1}\right), Q_{2}:=\left(x_{2}, y_{2}\right)$, and $Q_{3}:=\left(x_{3}, y_{3}\right)$ on $\mathbf{E}^{2}$, respectively, which form a triangle $\triangle Q_{1} Q_{2} Q_{3}$. Then, the invariants $F^{(2)}, F_{23}^{(2)}$ and $F_{13}^{(2)}$ are just, in this order, one half of the Euclidean distances $\overline{Q_{1} Q_{2}}, \overline{Q_{1} Q_{3}}$, and $\overline{Q_{2} Q_{3}}$. Meanwhile the area of the triangle $\triangle Q_{1} Q_{2} Q_{3}$ is just the constant $A$ in (2.14), which is, in fact, the Heron-Archimedes formula for the Euclidean area [29].

\section{Two-dimensional spaces of constant curvature}

This section provides the basic geometrical background to construct a $\left(\kappa_{1}, \kappa_{2}\right)$-parametric family of LH systems on curved spaces along with their invariants and superposition rules.

Let us consider a two-parametric family of 3D real Lie algebras, denoted by $\mathfrak{s o}_{\kappa_{1}, \kappa_{2}}(3)$, which depends on two real parameters, $\kappa_{1}$ and $\kappa_{2}$, which comprises the so-called $C K$ Lie algebras [23, 24, 25, 26, 27, 28, 29] or quasisimple orthogonal algebras [38]. The structure constants of $\mathfrak{s o}_{\kappa_{1}, \kappa_{2}}(3)$ in the basis $\left\{P_{1}, P_{2}, J_{12}\right\}$ are given by

$$
\left[J_{12}, P_{1}\right]=P_{2}, \quad\left[J_{12}, P_{2}\right]=-\kappa_{2} P_{1}, \quad\left[P_{1}, P_{2}\right]=\kappa_{1} J_{12} .
$$

The involutive automorphisms $\Theta_{0}, \Theta_{01}: \mathfrak{s o}_{\kappa_{1}, \kappa_{2}}(3) \rightarrow \mathfrak{s o}_{\kappa_{1}, \kappa_{2}}(3)$, defined by imposing

$$
\begin{array}{ccc}
\Theta_{0}\left(J_{12}\right) & =J_{12}, \quad \Theta_{0}\left(P_{1}\right)=-P_{1}, \quad \Theta_{0}\left(P_{2}\right)=-P_{2}, \\
\Theta_{01}\left(J_{12}\right) & =-J_{12}, \quad \Theta_{01}\left(P_{1}\right)=P_{1}, \quad \Theta_{01}\left(P_{2}\right)=-P_{2},
\end{array}
$$

diagonalize and commute among themselves. Hence, they induce a decomposition of $\mathfrak{s o}_{\kappa_{1}, \kappa_{2}}(3)$ into common eigenspaces of $\Theta_{0}$ and $\Theta_{01}$ of the form $\mathfrak{s o}_{\kappa_{1}, \kappa_{2}}(3)=E_{(1,0)} \oplus E_{(0,1)} \oplus E_{(1,1)}$, with $E_{(0,1)}:=\left\langle J_{12}\right\rangle$, $E_{(1,0)}:=\left\langle P_{1}\right\rangle, E_{(1,1)}:=\left\langle P_{2}\right\rangle$, and $E_{(0,0)}=\{0\}$. This gives rise to a $\mathbb{Z}_{2} \times \mathbb{Z}_{2}$-grading of the Lie algebra $\mathfrak{s o}_{\kappa_{1}, \kappa_{2}}(3)$, i.e. $\left[E_{\left(\alpha_{1}, \alpha_{2}\right)}, E_{\left(\beta_{1}, \beta_{2}\right)}\right] \subset E_{\left(\alpha_{1}+\beta_{1}, \alpha_{2}+\beta_{2}\right)}$ for every $\alpha_{1}, \alpha_{2}, \beta_{1}, \beta_{2} \in \mathbb{Z}_{2}$. Hence, $\kappa_{1}$ and $\kappa_{2}$ are two graded contraction parameters determined by $\Theta_{0}$ and $\Theta_{01}$, respectively [32. By rescaling the basis of $\mathfrak{s o}_{\kappa_{1}, \kappa_{2}}(3)$ each parameter $\kappa_{a}(a=1,2)$ can be reduced to either $+1,0$ or -1 . The vanishment of any $\kappa_{a}$ is equivalent to applying an Inönü-Wigner contraction [32].

The automorphism $\Theta_{0}$ gives rise to the Cartan decomposition:

$$
\mathfrak{s o}_{\kappa_{1}, \kappa_{2}}(3)=\mathfrak{h}_{0} \oplus \mathfrak{p}_{0}, \quad \mathfrak{h}_{0}:=\left\langle J_{12}\right\rangle \simeq \mathfrak{s o}_{\kappa_{2}}(2), \quad \mathfrak{p}_{0}:=\left\langle P_{1}, P_{2}\right\rangle,
$$


where $\mathfrak{s o}_{\kappa_{2}}(2)$ is the space of real $2 \times 2$ matrices $A$ satisfying that $A^{T} \mathbf{I}_{\kappa_{2}}+\mathbf{I}_{\kappa_{2}} A=0, \mathbf{I}_{\kappa_{2}}:=\operatorname{diag}\left(1, \kappa_{2}\right)$. The Lie algebra $\mathfrak{s o}_{\kappa_{1}, \kappa_{2}}(3)$ is isomorphic to the matrix Lie algebra of $3 \times 3$ real matrices $M$ satisfying [27.

$$
M^{T} \mathbf{I}_{\boldsymbol{\kappa}}+\mathbf{I}_{\boldsymbol{\kappa}} M=0, \quad \mathbf{I}_{\boldsymbol{\kappa}}:=\operatorname{diag}\left(1, \kappa_{1}, \kappa_{1} \kappa_{2}\right), \quad \boldsymbol{\kappa}:=\left(\kappa_{1}, \kappa_{2}\right) .
$$

If $\mathbf{I}_{\boldsymbol{\kappa}}$ is not degenerate, then this space is indeed the so-called indefinite orthogonal Lie algebra $\mathfrak{s o}(p, q)$, where $p$ and $q$ are the number of positive and negative eigenvalues of the matrix $\mathbf{I}_{\boldsymbol{\kappa}}$.

In particular, the elements of the basis $\left\{P_{1}, P_{2}, J_{12}\right\}$ can be identified with the matrices

$$
P_{1}=-\kappa_{1} e_{01}+e_{10}, \quad P_{2}=-\kappa_{1} \kappa_{2} e_{02}+e_{20}, \quad J_{12}=-\kappa_{2} e_{12}+e_{21},
$$

where $e_{i j}$ is the $3 \times 3$ matrix with a single non-zero entry 1 at row $i$ and column $j(i, j=0,1,2)$.

The elements of $\mathfrak{s o}_{\kappa_{1}, \kappa_{2}}(3)$ generate by matrix exponentiation the referred to as CK Lie group $\mathrm{SO}_{\kappa_{1}, \kappa_{2}}(3)$. The matrix exponentials of $\left\{P_{1}, P_{2}, J_{12}\right\}$ lead to the following one-parametric subgroups of the CK Lie group $\mathrm{SO}_{\kappa_{1}, \kappa_{2}}(3)$ :

$$
\begin{gathered}
\mathrm{e}^{\alpha P_{1}}=\left(\begin{array}{ccc}
\mathrm{C}_{\kappa_{1}}(\alpha) & -\kappa_{1} \mathrm{~S}_{\kappa_{1}}(\alpha) & 0 \\
\mathrm{~S}_{\kappa_{1}}(\alpha) & \mathrm{C}_{\kappa_{1}}(\alpha) & 0 \\
0 & 0 & 1
\end{array}\right), \\
\mathrm{e}^{\beta P_{2}}=\left(\begin{array}{ccc}
\mathrm{C}_{\kappa_{1} \kappa_{2}}(\beta) & 0 & -\kappa_{1} \kappa_{2} \mathrm{~S}_{\kappa_{1} \kappa_{2}}(\beta) \\
0 & 1 & 0 \\
\mathrm{~S}_{\kappa_{1} \kappa_{2}}(\beta) & 0 & \mathrm{C}_{\kappa_{1} \kappa_{2}}(\beta)
\end{array}\right),
\end{gathered}
$$

where the so-called $\kappa$-dependent cosine and sine functions read [27, 28, 29]:

$$
\begin{gathered}
\mathrm{C}_{\kappa}(u):=\sum_{l=0}^{\infty}(-\kappa)^{l} \frac{u^{2 l}}{(2 l) !}=\left\{\begin{array}{cc}
\cos \sqrt{\kappa} u & \kappa>0 \\
1 & \kappa=0 \\
\operatorname{ch} \sqrt{-\kappa} u & \kappa<0
\end{array}\right. \\
\mathrm{S}_{\kappa}(u):=\sum_{l=0}^{\infty}(-\kappa)^{l} \frac{u^{2 l+1}}{(2 l+1) !}=\left\{\begin{array}{cc}
\frac{1}{\sqrt{\kappa}} \sin \sqrt{\kappa} u & \kappa>0 \\
u & \kappa=0 \\
\frac{1}{\sqrt{-\kappa}} \operatorname{sh} \sqrt{-\kappa} u & \kappa<0
\end{array} .\right.
\end{gathered}
$$

From them, the $\kappa$-tangent and the $\kappa$-versed sine (or versine) take the form

$$
\mathrm{T}_{\kappa}(u):=\frac{\mathrm{S}_{\kappa}(u)}{\mathrm{C}_{\kappa}(u)}, \quad \mathrm{V}_{\kappa}(u):=\frac{1}{\kappa}\left(1-\mathrm{C}_{\kappa}(u)\right) .
$$

These $\kappa$-functions cover both the usual circular $(\kappa>0)$ and hyperbolic $(\kappa<0)$ trigonometric functions. In the case $\kappa=0$, the previous functions reduce to the parabolic ones $\mathrm{C}_{0}(u)=1, \mathrm{~S}_{0}(u)=\mathrm{T}_{0}(u)=u$, and $\mathrm{V}_{0}(u)=u^{2} / 2$.

Some relations for the above $\kappa$-functions read

$$
\mathrm{C}_{\kappa}^{2}(u)+\kappa \mathrm{S}_{\kappa}^{2}(u)=1, \quad \mathrm{C}_{\kappa}(2 u)=\mathrm{C}_{\kappa}^{2}(u)-\kappa \mathrm{S}_{\kappa}^{2}(u), \quad \mathrm{S}_{\kappa}(2 u)=2 \mathrm{~S}_{\kappa}(u) \mathrm{C}_{\kappa}(u),
$$

and their derivatives are given by

$$
\frac{\mathrm{d}}{\mathrm{d} u} \mathrm{C}_{\kappa}(u)=-\kappa \mathrm{S}_{\kappa}(u), \quad \frac{\mathrm{d}}{\mathrm{d} u} \mathrm{~S}_{\kappa}(u)=\mathrm{C}_{\kappa}(u), \quad \frac{\mathrm{d}}{\mathrm{d} u} \mathrm{~T}_{\kappa}(u)=\frac{1}{\mathrm{C}_{\kappa}^{2}(u)}, \quad \frac{\mathrm{d}}{\mathrm{d} u} \mathrm{~V}_{\kappa}(u)=\mathrm{S}_{\kappa}(u) .
$$

Many other relations can be found in 29].

Let $H_{0}:=\mathrm{SO}_{\kappa_{2}}(2)$ be the Lie subgroup of $\mathrm{SO}_{\kappa_{1}, \kappa_{2}}(3)$ obtained by matrix exponentiation of the Lie algebra $\mathfrak{h}_{0}$. The CK family of $2 \mathrm{D}$ homogeneous spaces is defined by the quotient

$$
\mathbf{S}_{\left[\kappa_{1}\right], \kappa_{2}}^{2}:=\mathrm{SO}_{\kappa_{1}, \kappa_{2}}(3) / \mathrm{SO}_{\kappa_{2}}(2) .
$$

The (possibly degenerate) metric defined by $\mathbf{I}_{\boldsymbol{\kappa}}$ (3.2) on $T_{e} \mathrm{SO}_{\kappa_{1}, \kappa_{2}}(3) \simeq \mathfrak{s o}_{\kappa_{1}, \kappa_{2}}(3)$ can be extended by right translation to a metric on the whole $S O_{\kappa_{1}, \kappa_{2}}(3)$ and then projected onto $\mathbf{S}_{\left[\kappa_{1}\right], \kappa_{2}}^{2}$. Then, the CK family becomes a symmetric space relative to the obtained metric. The contraction parameter $\kappa_{1}$ becomes the constant (Gaussian) curvature of the space. The second parameter $\kappa_{2}$ determines the signature of the metric through $\operatorname{diag}\left(+, \kappa_{2}\right)$. 


\subsection{Ambient, geodesic parallel and geodesic polar coordinates}

The matrix realization (3.4) enables us to identify the elements of $\mathrm{SO}_{\kappa_{1}, \kappa_{2}}(3)$ with isometries of the bilinear form $\mathbf{I}_{\boldsymbol{\kappa}}(3.2)$. More specifically, given a $3 \times 3$ matrix $g$, it follows that

$$
g \in \mathrm{SO}_{\kappa_{1}, \kappa_{2}}(3) \Rightarrow g^{T} \mathbf{I}_{\boldsymbol{\kappa}} g=\mathbf{I}_{\boldsymbol{\kappa}} .
$$

This allows us to consider the Lie group action of $\mathrm{SO}_{\kappa_{1}, \kappa_{2}}(3)$ on $\mathbb{R}^{3}$ as isometries of $\mathbf{I}_{\boldsymbol{\kappa}}$.

The subgroup $\mathrm{SO}_{\kappa_{2}}(2)=\left\langle\mathrm{e}^{\gamma J_{12}}\right\rangle$ is the isotropy subgroup of the point $O:=(1,0,0)$, which is taken as the origin in the space $\mathbf{S}_{\left[\kappa_{1}\right], \kappa_{2}}^{2}$. Hence, $\mathrm{SO}_{\kappa_{1}, \kappa_{2}}(3)$ becomes an isometry group of the space $\mathbf{S}_{\left[\kappa_{1}\right], \kappa_{2}}^{2}$, in such a manner that $J_{12}$ is a rotation generator, while $P_{1}$ and $P_{2}$ move $O$ along two basic geodesics $l_{1}$ and $l_{2}$, which are orthogonal at $O$, so behaving as translation generators (see figure 1).

The orbit of $O$ is contained in the submanifold given by $\mathbf{I}_{\boldsymbol{\kappa}}$ of the form

$$
\Sigma_{\boldsymbol{\kappa}}:=\left\{v:=\left(x_{0}, x_{1}, x_{2}\right) \in \mathbb{R}^{3}: \mathbf{I}_{\boldsymbol{\kappa}}(v, v)=x_{0}^{2}+\kappa_{1} x_{1}^{2}+\kappa_{1} \kappa_{2} x_{2}^{2}=1\right\} .
$$

This orbit, namely the connected component of $\Sigma_{\kappa}$ containing the point $O$, can be identified with the space $\mathbf{S}_{\left[\kappa_{1}\right], \kappa_{2}}^{2}$. The coordinates $\left\{x_{0}, x_{1}, x_{2}\right\}$ on $\mathbb{R}^{3}$, satisfying the constraint (3.8) on $\Sigma_{\kappa}$, are called ambient or Weierstrass coordinates. In these variables, the metric on $\mathbf{S}_{\left[\kappa_{1}\right], \kappa_{2}}^{2}$ comes from the flat ambient metric in $\mathbb{R}^{3}$ divided by the curvature $\kappa_{1}$ and restricted to $\Sigma_{\kappa}$, namely

$$
\mathrm{d} s_{\kappa}^{2}:=\left.\frac{1}{\kappa_{1}}\left(\mathrm{~d} x_{0}^{2}+\kappa_{1} \mathrm{~d} x_{1}^{2}+\kappa_{1} \kappa_{2} \mathrm{~d} x_{2}^{2}\right)\right|_{\Sigma_{\kappa}}=\frac{\kappa_{1}\left(x_{1} \mathrm{~d} x_{1}+\kappa_{2} x_{2} \mathrm{~d} x_{2}\right)^{2}}{1-\kappa_{1} x_{1}^{2}-\kappa_{1} \kappa_{2} x_{2}^{2}}+\mathrm{d} x_{1}^{2}+\kappa_{2} \mathrm{~d} x_{2}^{2} .
$$

It is worth noting that if $\kappa_{1}=0$, then $\Sigma_{\kappa}$ is given by two connected components with $x_{0} \in\{-1,1\}$ and $\mathrm{d} s_{\kappa}^{2}$ is well-defined.

The ambient coordinates can be parametrized on $\Sigma_{\kappa}$ in terms of two intrinsic variables in different ways (see e.g. [28, 39]). In particular, let us introduce the so-called geodesic parallel $\{x, y\}$ and geodesic polar $\{r, \phi\}$ coordinates of a point $Q:=\left(x_{0}, x_{1}, x_{2}\right)$ in $\mathbf{S}_{\left[\kappa_{1}\right], \kappa_{2}}^{2}$ which are obtained through the following action of the one-parametric subgroups (3.4) on $O[28]$ :

$$
\left(x_{0}, x_{1}, x_{2}\right)^{T}=\exp \left(x P_{1}\right) \exp \left(y P_{2}\right) O^{T}=\exp \left(\phi J_{12}\right) \exp \left(r P_{1}\right) O^{T}
$$

yielding

$$
\begin{aligned}
& x_{0}=\mathrm{C}_{\kappa_{1}}(x) \mathrm{C}_{\kappa_{1} \kappa_{2}}(y)=\mathrm{C}_{\kappa_{1}}(r), \\
& x_{1}=\mathrm{S}_{\kappa_{1}}(x) \mathrm{C}_{\kappa_{1} \kappa_{2}}(y)=\mathrm{S}_{\kappa_{1}}(r) \mathrm{C}_{\kappa_{2}}(\phi), \\
& x_{2}=\mathrm{S}_{\kappa_{1} \kappa_{2}}(y)=\mathrm{S}_{\kappa_{1}}(r) \mathrm{S}_{\kappa_{2}}(\phi) .
\end{aligned}
$$

By introducing these relations in the metric (3.9) and applying (3.6), we recover the usual (curved) metrics given by

$$
\mathrm{d} s_{\kappa}^{2}=\mathrm{C}_{\kappa_{1} \kappa_{2}}^{2}(y) \mathrm{d} x^{2}+\kappa_{2} \mathrm{~d} y^{2}=\mathrm{d} r^{2}+\kappa_{2} \mathrm{~S}_{\kappa_{1}}^{2}(r) \mathrm{d} \phi^{2} .
$$

As shown in figure 1, the variable $r$ is the distance between the origin $O$ and the point $Q$ measured along the geodesic $l$ that joins both points, while $\phi$ is the angle of $l$ relative to basic geodesic $l_{1}$. If $Q_{1}$ denotes the intersection point of $l_{1}$ with its orthogonal geodesic $l_{2}^{\prime}$ through $Q$, then $x$ is the geodesic distance between $O$ and $Q_{1}$ measured along $l_{1}$ and $y$ is the geodesic distance between $Q_{1}$ and $Q$ measured along $l_{2}^{\prime}$. Note that a second set of geodesic parallel coordinates $\left\{x^{\prime}, y^{\prime}\right\}$, similar to $\{x, y\}$, can also be defined by considering the intersection point $Q_{2}$ of $l_{2}$ with its orthogonal geodesic $l_{1}^{\prime}$ through $Q$, and that $\{x, y\} \neq\left\{x^{\prime}, y^{\prime}\right\}$ if the curvature $\kappa_{1} \neq 0\left[28\right.$. On the flat Euclidean plane $\mathbf{E}^{2}$ with $\kappa_{1}=0,\{x, y\}=\left\{x^{\prime}, y^{\prime}\right\}$ reduce to Cartesian coordinates and $\{r, \phi\}$ to the usual polar ones.

Since we are interested in extending the Euclidean $\mathrm{P}_{1}$-LH systems of section 2 to all the CK spaces, we shall make use of the geodesic parallel coordinates $(x, y)$, although the relations (3.10) would enable one to express our final results in terms of the geodesic polar ones.

Summing up, according to the values of the two $\kappa_{a}$ parameters, the CK space $\mathbf{S}_{\left[\kappa_{1}\right], \kappa_{2}}^{2}$ comprises nine specific 2D symmetrical homogeneous spaces, which depending on the parameter $\kappa_{2}$ are classified into three types: 


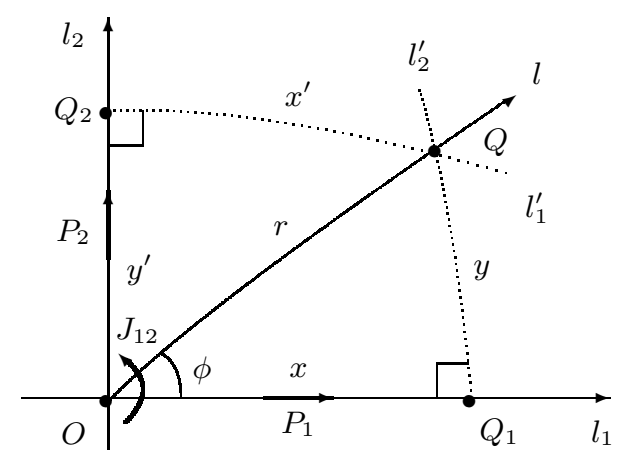

Figure 1: Schematic representation of the isometry infinitesimal generators $\left\{J_{12}, P_{1}, P_{2}\right\}$ and geodesic coordinates $\{x, y\},\left\{x^{\prime}, y^{\prime}\right\}$ and $\{r, \phi\}$ of a point $Q=\left(x_{0}, x_{1}, x_{2}\right)$ on a $2 \mathrm{D}$ CK space.

- Riemannian spaces for $\kappa_{2}>0$. The standard sphere $\mathbf{S}^{2}$ arises when $\kappa_{1}>0$. The case $\kappa_{1}<0$ leads to a two-sheeted hyperboloid. We call $\mathbf{H}^{2}$ the upper sheet of the hyperboloid, namely the part with $x_{0} \geq 1$ : the so-called Lobachevsky space. The contraction $\kappa_{1}=0$ gives rise to two Euclidean planes $x_{0}= \pm 1$. We will call Euclidean space, $\mathbf{E}^{2}$, the one with $x_{0}=+1$.

- Pseudo-Riemannian spaces or Lorentzian spacetimes for $\kappa_{2}<0$. For Gaussian curvature $\kappa_{1}>0$, we obtain the $2 D$ co-hyperbolic space or $(1+1) D$ anti-de Sitter spacetime $\mathbf{A d S}^{\mathbf{1 + 1}}$; if $\kappa_{1}<0$, we find the $2 D$ doubly-hyperbolic space or $(1+1) D$ de Sitter spacetime $\mathbf{d S}^{\mathbf{1 + 1}}$; and the flat case with $\kappa_{1}=0$ provides the $(1+1) \mathrm{D}$ Minkowskian spacetime $\mathbf{M}^{\mathbf{1}+\mathbf{1}}$. In all cases for $\kappa_{2}<0$, the $J_{12}, P_{1}$, and $P_{2}$ correspond to the infinitesimal generators of boosts, time translations, and spatial translations, respectively. From a physic viewpoint, the $\kappa_{a}$ parameters are related to the cosmological constant $\Lambda$ and the speed of light $c$ through

$$
\kappa_{1}=-\Lambda, \quad \kappa_{2}=-1 / c^{2} .
$$

And the geodesic parallel coordinates $(x, y)$ are just the time $t$ and space $y$ ones.

- Semi-Riemannian spaces or Newtonian spacetimes for $\kappa_{2}=0(c=\infty)$. In this case, the metric $(3.9)$ is degenerate and the kernel of the metric gives rise to an integrable foliation of $\mathbf{S}_{\left[\kappa_{1}\right], 0}^{2}$, which is invariant under the action of the CK group $\mathrm{SO}_{\kappa_{1}, 0}(3)$ on $\mathbf{S}_{\left[\kappa_{1}\right], 0}^{2}$. There appears a well-defined subsidiary metric $\mathrm{d}{s^{\prime}}^{2}:=\mathrm{d} s_{\kappa}^{2} / \kappa_{2}$ restricted to each leaf, which in the coordinates $(x, y)$ read [28]

$$
\mathrm{d} s^{2}=\mathrm{d} x^{2}, \quad \mathrm{~d} s^{\prime 2}=\mathrm{d} y^{2} \quad \text { on } \quad x=\text { constant. }
$$

For $\kappa_{1}>0$ we find the $2 D$ co-Euclidean space or $(1+1) D$ oscillating Newton-Hook (NH) spacetime $\mathbf{N H}_{+}^{\mathbf{1 + 1}}$, and for $\kappa_{1}<0$ we obtain the 2D co-Minkowskian space or $(1+1) D$ expanding NH spacetime $\mathbf{N H}_{-}^{\mathbf{1 + 1}}$. The flat space with $\kappa_{1}=0$ is just the Galilean one $\mathbf{G}^{\mathbf{1 + 1}}$. Hence, in these three cases, the metric $\mathrm{d} s_{\kappa}^{2}$ provides 'absolute-time' $t$, the leaves of the invariant foliation are the 'absolute-space' at $t=t_{0}$ and $\mathrm{d} s_{\kappa}^{\prime 2}$ is the subsidiary spatial metric defined on each leaf.

Each specific CK space, Lie algebra of infinitesimal symmetries, and metric are displayed in table 1 in the next section.

\section{A class of LH systems on curved spaces}

We shall hereafter make extensive use of the shorthand notation $\boldsymbol{\kappa}:=\left(\kappa_{1}, \kappa_{2}\right)$. Our procedure consists in defining a Lie system $X_{\kappa}$ possessing a Vessiot-Guldberg Lie algebra $V_{\kappa}$ consisting of infinitesimal symmetries of the metric of the CK space $\mathbf{S}_{\left[\kappa_{1}\right], \kappa_{2}}^{2}$. Next, we obtain a compatible symplectic form $\omega_{\kappa}$ turning the elements of $V_{\kappa}$ into Hamiltonian vector fields. 
The fundamental vector fields of the Lie group action of $\mathrm{SO}_{\boldsymbol{\kappa}}(3)$ on $\mathbb{R}^{3}$ by isometries of $\mathbf{I}_{\boldsymbol{\kappa}}$ are Lie symmetries of $\mathrm{d} s_{\boldsymbol{\kappa}}^{2}$. Since the action is linear, the fundamental vector fields can be obtained straightforwardly from the 3D matrix representation (3.3). In ambient coordinates $\left(x_{0}, x_{1}, x_{2}\right)$, they read [28],

$$
P_{1}:=\kappa_{1} x_{1} \frac{\partial}{\partial x_{0}}-x_{0} \frac{\partial}{\partial x_{1}}, \quad P_{2}:=\kappa_{1} \kappa_{2} x_{2} \frac{\partial}{\partial x_{0}}-x_{0} \frac{\partial}{\partial x_{2}}, \quad J_{12}:=\kappa_{2} x_{2} \frac{\partial}{\partial x_{1}}-x_{1} \frac{\partial}{\partial x_{2}} .
$$

Since the function $\mathbf{I}_{\boldsymbol{\kappa}}(v, v)=x_{0}^{2}+\kappa_{1} x_{1}^{2}+\kappa_{1} \kappa_{2} x_{2}^{2}$ is an invariant of the action of the Lie group action $\mathrm{SO}_{\kappa}(3)$, the above vector fields can be restricted to $\Sigma_{\kappa}$. Such restrictions are Lie symmetries of the restriction of $\mathrm{d} s_{\boldsymbol{\kappa}}^{2}$. These vector fields are the 'curved' counterpart of the initial Euclidean ones $X_{i}$ (2.4) in any coordinate system. In terms of geodesic parallel coordinates (3.10) and using (3.6), they become

$$
\begin{gathered}
X_{\kappa, 1}:=-P_{1}=\frac{\partial}{\partial x}, \quad X_{\kappa, 2}:=-P_{2}=\kappa_{1} \kappa_{2} \mathrm{~S}_{\kappa_{1}}(x) \mathrm{T}_{\kappa_{1} \kappa_{2}}(y) \frac{\partial}{\partial x}+\mathrm{C}_{\kappa_{1}}(x) \frac{\partial}{\partial y}, \\
X_{\kappa, 3}:=J_{12}=\kappa_{2} \mathrm{C}_{\kappa_{1}}(x) \mathrm{T}_{\kappa_{1} \kappa_{2}}(y) \frac{\partial}{\partial x}-\mathrm{S}_{\kappa_{1}}(x) \frac{\partial}{\partial y} .
\end{gathered}
$$

Then the $t$-dependent vector field

$$
X_{\kappa}:=b_{1}(t) X_{\kappa, 1}+b_{2}(t) X_{\kappa, 2}+b_{3}(t) X_{\kappa, 3},
$$

provides the following system of nonautonomous differential equations

$$
\begin{aligned}
& \frac{\mathrm{d} x}{\mathrm{~d} t}=b_{1}(t)+\kappa_{1} \kappa_{2} b_{2}(t) \mathrm{S}_{\kappa_{1}}(x) \mathrm{T}_{\kappa_{1} \kappa_{2}}(y)+\kappa_{2} b_{3}(t) \mathrm{C}_{\kappa_{1}}(x) \mathrm{T}_{\kappa_{1} \kappa_{2}}(y), \\
& \frac{\mathrm{d} y}{\mathrm{~d} t}=b_{2}(t) \mathrm{C}_{\kappa_{1}}(x)-b_{3}(t) \mathrm{S}_{\kappa_{1}}(x) .
\end{aligned}
$$

Obviously, $X_{\kappa}$ is a Lie system and the vector fields (4.1) satisfy the commutation relations (3.1), that is,

$$
\left[X_{\kappa, 3,}, X_{\kappa, 1}\right]=X_{\kappa, 2}, \quad\left[X_{\kappa, 3} X_{\kappa, 2}\right]=-\kappa_{2} X_{\kappa, 1}, \quad\left[X_{\kappa, 1}, X_{\kappa, 2}\right]=\kappa_{1} X_{\kappa, 3},
$$

so spanning a Vessiot-Guldberg Lie algebra $V_{\boldsymbol{\kappa}}$ isomorphic to the CK Lie algebra $\mathfrak{s o}_{\kappa}(3)$. If we now consider the Euclidean space $\mathbf{E}^{2}$ with parameters $\boldsymbol{\kappa}=\left(\kappa_{1}, \kappa_{2}\right)=(0,+1)$, we find that vector fields (4.1), differential equations (4.3), and commutation rules (4.4) reduce to (2.4), (2.5) and (2.6), respectively.

Furthermore, the restriction of the vector fields $X_{\kappa, i}$ to $\Sigma_{\kappa}$ can be turned into Hamiltonian vector fields with Hamiltonian functions $h_{\kappa, i}$ with respect to a symplectic form $\omega_{\kappa}$. Recall that they are infinitesimal symmetries of $\mathrm{ds}_{\kappa}^{2}$. If ds $\mathrm{s}_{\kappa}^{2}$ is not degenerate, then $V_{\kappa}$ becomes a Lie algebra of Killing vector fields relative to $\mathrm{ds}_{\kappa}^{2}$. Hence, they are Lie symmetries of the volume form $\omega_{\kappa}$ on $\Sigma_{\kappa}$ induced by $\mathrm{ds}_{\kappa}^{2}$. Up to a non-zero proportional constant,

$$
\omega_{\kappa}=\mathrm{C}_{\kappa_{1} \kappa_{2}}(y) \mathrm{d} x \wedge \mathrm{d} y .
$$

The case when $\mathrm{d} s_{\boldsymbol{\kappa}}^{2}$ is degenerate can be obtained by making an appropriate limit in $\boldsymbol{\kappa}$. Obviously, $\omega_{\boldsymbol{\kappa}}$ is the area element $\mathrm{d} A$ for all the CK spaces [28.

Next, the relation $\iota_{X_{\kappa}, i} \omega_{\kappa}=\mathrm{d} h_{\kappa, i}$ allows us to determine some Hamiltonian functions $h_{\kappa, i}$ for the vector fields $X_{\kappa, i}$ with respect to the symplectic form $\omega_{\kappa}$ :

$$
\begin{gathered}
h_{\kappa, 1}=\mathrm{S}_{\kappa_{1} \kappa_{2}}(y), \quad h_{\kappa, 2}=-\mathrm{S}_{\kappa_{1}}(x) \mathrm{C}_{\kappa_{1} \kappa_{2}}(y), \\
h_{\kappa, 3}=\frac{1}{\kappa_{1}}\left(1-\mathrm{C}_{\kappa_{1}}(x) \mathrm{C}_{\kappa_{1} \kappa_{2}}(y)\right)=\mathrm{V}_{\kappa_{1}}(x)+\kappa_{2} \mathrm{~V}_{\kappa_{1} \kappa_{2}}(y)-\kappa_{1} \kappa_{2} \mathrm{~V}_{\kappa_{1}}(x) \mathrm{V}_{\kappa_{1} \kappa_{2}}(y) .
\end{gathered}
$$

The above functions span, along with a function $h_{\kappa, 0}=1$, a Lie algebra of functions relative to the Poisson bracket $\{\cdot, \cdot\}_{\omega_{\kappa}}$ induced by $\omega_{\kappa}$. In fact, the base of such a Lie algebra satisfies the following commutation relations

$$
\begin{gathered}
\left\{h_{\boldsymbol{\kappa}, 3}, h_{\boldsymbol{\kappa}, 1}\right\}_{\omega_{\boldsymbol{\kappa}}}=-h_{\boldsymbol{\kappa}, 2}, \quad\left\{h_{\boldsymbol{\kappa}, 3}, h_{\boldsymbol{\kappa}, 2}\right\}_{\omega_{\boldsymbol{\kappa}}}=\kappa_{2} h_{\boldsymbol{\kappa}, 1}, \\
\left\{h_{\boldsymbol{\kappa}, 1}, h_{\boldsymbol{\kappa}, 2}\right\}_{\omega_{\boldsymbol{\kappa}}}=h_{\boldsymbol{\kappa}, 0}-\kappa_{1} h_{\boldsymbol{\kappa}, 3}, \quad\left\{h_{\boldsymbol{\kappa}, 0}, \cdot\right\}_{\omega_{\boldsymbol{\kappa}}}=0 .
\end{gathered}
$$


Indeed, $h_{\boldsymbol{\kappa}, 0}$ is a central generator in such a manner that $\left(\left\langle h_{\kappa, 1}, h_{\kappa, 2}, h_{\kappa, 3}, h_{\kappa, 0}\right\rangle,\{\cdot, \cdot\}_{\omega_{\kappa}}\right)$ span a LH algebra $\mathcal{H}_{\omega_{\kappa}}$ which is isomorphic to a central extension of the CK Lie algebra $\mathfrak{s o}_{\kappa}(3)$, denoted by $\overline{\mathfrak{s o}}_{\kappa}(3)$. In this way, we obtain the $t$-dependent Hamiltonian associated with the Lie system (4.2):

$$
h_{\boldsymbol{\kappa}}(t)=b_{1}(t) h_{\kappa, 1}+b_{2}(t) h_{\kappa, 2}+b_{3}(t) h_{\kappa, 3} .
$$

We remark that the addition of a central generator $h_{\kappa, 0}$ is necessary to ensure that the Hamiltonian functions $h_{\kappa, i}$ span a Lie algebra, similarly to the Euclidean case described in section 2. However, it is well-known that the central extension is trivial when $\kappa_{1} \neq 0$ [40. This, in turn, means that if we apply the change of basis

$$
h_{\boldsymbol{\kappa}, 1}^{\prime}=h_{\boldsymbol{\kappa}, 1}, \quad h_{\boldsymbol{\kappa}, 2}^{\prime}=h_{\boldsymbol{\kappa}, 2}, \quad h_{\boldsymbol{\kappa}, 3}^{\prime}=h_{\boldsymbol{\kappa}, 3}-h_{\boldsymbol{\kappa}, 0} / \kappa_{1}, \quad \kappa_{1} \neq 0,
$$

the trivial extension is 'removed' and the commutation relations (4.7) become

$$
\left\{h_{\boldsymbol{\kappa}, 3}^{\prime}, h_{\boldsymbol{\kappa}, 1}^{\prime}\right\}_{\omega_{\kappa}}=-h_{\boldsymbol{\kappa}, 2}^{\prime}, \quad\left\{h_{\boldsymbol{\kappa}, 3}^{\prime}, h_{\boldsymbol{\kappa}, 2}^{\prime}\right\}_{\omega_{\kappa}}=\kappa_{2} h_{\boldsymbol{\kappa}, 1}^{\prime} \quad\left\{h_{\boldsymbol{\kappa}, 1}^{\prime}, h_{\boldsymbol{\kappa}, 2}^{\prime}\right\}_{\omega_{\kappa}}=-\kappa_{1} h_{\boldsymbol{\kappa}, 3}^{\prime},
$$

which are just the commutation relations (4.4) of the CK Lie algebra $\mathfrak{s o}_{\kappa}(3)$ for $\kappa_{1} \neq 0$. In this case, the LH algebra $\mathcal{H}_{\omega_{\kappa}} \simeq \overline{\mathfrak{s o}}_{\kappa}(3) \simeq \mathfrak{s o}_{\kappa}(3) \oplus \mathbb{R}$. On the contrary, if $\kappa_{1}=0$ the central extension $h_{\kappa, 0}$ is a non-trivial one 40] (this cannot be 'removed' through a change of basis) and the commutation rules (4.7) read

$$
\left\{h_{\boldsymbol{\kappa}, 3}, h_{\boldsymbol{\kappa}, 1}\right\}_{\omega_{\boldsymbol{\kappa}}}=-h_{\boldsymbol{\kappa}, 2}, \quad\left\{h_{\boldsymbol{\kappa}, 3}, h_{\boldsymbol{\kappa}, 2}\right\}_{\omega_{\boldsymbol{\kappa}}}=\kappa_{2} h_{\boldsymbol{\kappa}, 1}, \quad\left\{h_{\boldsymbol{\kappa}, 1}, h_{\boldsymbol{\kappa}, 2}\right\}_{\omega_{\boldsymbol{\kappa}}}=h_{\boldsymbol{\kappa}, 0}, \quad\left\{h_{\boldsymbol{\kappa}, 0}, \cdot\right\}_{\omega_{\boldsymbol{\kappa}}}=0,
$$

which correspond to central extensions of non-simple Lie algebras: Euclidean $\overline{\mathfrak{i} \mathfrak{s o}}(2) \simeq \overline{\mathfrak{s o}(2) \ltimes \mathbb{R}^{2}}\left(\kappa_{2}>0\right)$ (so recovering (2.8)), Poincaré $\overline{\mathfrak{i s o}}(1,1) \simeq \overline{\mathfrak{s o}(1,1) \ltimes \mathbb{R}^{2}}\left(\kappa_{2}<0\right)$, and Galilei $\overline{\mathfrak{i i s o}}(1) \simeq \overline{\mathbb{R} \ltimes \mathbb{R}^{2}}\left(\kappa_{2}=0\right)$.

Notice also that the Hamiltonian function $h_{\kappa, 3}$ (4.6) is written in two forms. The former requires to take the limit $\kappa_{1} \rightarrow 0$ for the flat cases taking power series of $\mathrm{C}_{\kappa}(u)$, but the latter (in terms of $\kappa$-versed sines (3.5)) directly provides the same result by setting $\kappa_{1}=0$.

We display in table 1 the specific vector fields (4.1), Hamiltonian functions (4.6), and symplectic form (4.5) for each of the nine spaces comprised within the CK family (3.7).

\section{Constants of motion and superposition rules}

This section deals with the computations of the constants of motion for the LH system $X_{\kappa}$ (4.2), which will further allow us to deduce the corresponding superposition rules by applying the Poisson coalgebra approach [9, 13].

\subsection{Constants of motion}

Likewise in section 2.1, the space $S\left(\overline{\mathfrak{s o}}_{\kappa}(3)\right)$ stands for the symmetric algebra of the extended CK Lie algebra $\overline{\mathfrak{s o}}_{\kappa}(3)$. The symmetric algebra is naturally a Poisson algebra. Consider a basis $\left\{v_{1}, v_{2}, v_{3}, v_{0}\right\}$ of $\overline{\mathfrak{s o}}_{\kappa}(3)$ satisfying the commutation relations (4.7). Then, the element

$$
C_{\kappa}:=v_{3} v_{0}-\frac{1}{2}\left(\kappa_{2} v_{1}^{2}+v_{2}^{2}+\kappa_{1} v_{3}^{2}\right)
$$

Poisson commutes with all $v_{a}$, i.e. it is a second-order Casimir (invariant) of $S\left(\overline{\mathfrak{s o}}_{\kappa}(3)\right)$. Next, we consider the non-deformed coproduct map $\Delta: S\left(\overline{\mathfrak{s o}}_{\kappa}(3)\right) \rightarrow S\left(\overline{\mathfrak{s o}}_{\kappa}(3)\right) \otimes S\left(\overline{\mathfrak{s o}}_{\kappa}(3)\right)$ given by (2.9) along with the Poisson algebra morphisms $D: S\left(\overline{\mathfrak{s o}}_{\kappa}(3)\right) \rightarrow C^{\infty}\left(\mathbf{S}_{\left[\kappa_{1}\right], \kappa_{2}}^{2}\right)$ and $D^{(2)}: S\left(\overline{\mathfrak{s o}}_{\kappa}(3)\right) \otimes S\left(\overline{\mathfrak{s o}}_{\kappa}(3)\right) \rightarrow$ $C^{\infty}\left(\mathbf{S}_{\left[\kappa_{1}\right], \kappa_{2}}^{2}\right) \otimes C^{\infty}\left(\mathbf{S}_{\left[\kappa_{1}\right], \kappa_{2}}^{2}\right)$ defined, similarly to (2.10), by

$$
D\left(v_{a}\right):=h_{\boldsymbol{\kappa}, a}\left(x_{1}, y_{1}\right), \quad D^{(2)}\left(v_{a} \otimes 1\right):=h_{\kappa, a}\left(x_{1}, y_{1}\right), \quad D^{(2)}\left(1 \otimes v_{a}\right):=h_{\boldsymbol{\kappa}, a}\left(x_{2}, y_{2}\right),
$$


Table 1: LH algebras on the nine CK spaces according to the 'normalized' values of the contraction parameters $\kappa_{a} \in\{1,0,-1\}$. For each space $\mathbf{S}_{\left[\kappa_{1}\right], \kappa_{2}}^{2}$ (3.7) it is shown, in geodesic parallel coordinates $(x, y)$ (3.10), the metric $\mathrm{d} s_{\kappa}^{2}$ (3.11), domain of the variables, Vessiot-Guldberg Lie algebra $V_{\boldsymbol{\kappa}}$ (4.4) with Lie vector fields $X_{\kappa, i}$ (4.1), LH algebra $\mathcal{H}_{\omega_{\kappa}}$ (4.7) with Hamiltonian functions $h_{\kappa, i}$ (4.6) (so $h_{\kappa, 0}=1$ ), and the symplectic form $\omega_{\kappa}$ (4.5). For the sake of clarity, we drop the index $\boldsymbol{\kappa}=\left(\kappa_{1}, \kappa_{2}\right)$.

\begin{tabular}{|c|c|c|}
\hline - Sphere $\mathbf{S}^{2}$ & - Euclidean plane $\mathbf{E}^{2}$ & - Hyperbolic space $\mathbf{H}^{2}$ \\
\hline $\mathbf{S}_{[+],+}^{2}=\mathrm{SO}(3) / \mathrm{SO}(2)$ & $\mathbf{S}_{[0],+}^{2}=\operatorname{ISO}(2) / \mathrm{SO}(2)$ & $\mathbf{S}_{[-],+}^{2}=\mathrm{SO}(2,1) / \mathrm{SO}(2)$ \\
\hline $\mathrm{d} s^{2}=\cos ^{2} y \mathrm{~d} x^{2}+\mathrm{d} y^{2}$ & $\mathrm{~d} s^{2}=\mathrm{d} x^{2}+\mathrm{d} y^{2}$ & $\mathrm{~d} s^{2}=\cosh ^{2} y \mathrm{~d} x^{2}+\mathrm{d} y^{2}$ \\
\hline$x \in(-\pi, \pi], y \in\left(-\frac{\pi}{2}, \frac{\pi}{2}\right]$ & $x \in \mathbb{R}, y \in \mathbb{R}$ & $x \in \mathbb{R}, y \in \mathbb{R}$ \\
\hline$V \simeq \mathfrak{s o}(3)$ & $V \simeq \mathfrak{i s o}(2) \simeq \mathfrak{s o}(2) \ltimes \mathbb{R}^{2}$ & $V \simeq \mathfrak{s o}(2,1)$ \\
\hline$X_{1}=\partial_{x}$ & $X_{1}=\partial_{x}$ & $X_{1}=\partial_{x}$ \\
\hline$X_{2}=\sin x \tan y \partial_{x}+\cos x \partial_{y}$ & $X_{2}=\partial_{y}$ & $X_{2}=-\sinh x \tanh y \partial_{x}+\cosh x \partial_{y}$ \\
\hline$X_{3}=\cos x \tan y \partial_{x}-\sin x \partial_{y}$ & $X_{3}=y \partial_{x}-x \partial_{y}$ & $X_{3}=\cosh x \tanh y \partial_{x}-\sinh x \partial_{y}$ \\
\hline $\mathcal{H}_{\omega} \simeq \overline{\mathfrak{s o}}(3) \simeq \mathfrak{s o}(3) \oplus \mathbb{R}$ & $\mathcal{H}_{\omega} \simeq \overline{\mathfrak{i s o}}(2)=\overline{\mathfrak{s o}(2) \ltimes \mathbb{R}^{2}}$ & $\mathcal{H}_{\omega} \simeq \overline{\mathfrak{s o}}(2,1) \simeq \mathfrak{s o}(2,1) \oplus \mathbb{R}$ \\
\hline$h_{1}=\sin y$ & $h_{1}=y$ & $h_{1}=\sinh y$ \\
\hline$h_{2}=-\sin x \cos y$ & $h_{2}=-x$ & $h_{2}=-\sinh x \cosh y$ \\
\hline$h_{3}=1-\cos x \cos y$ & $h_{3}=\frac{1}{2}\left(x^{2}+y^{2}\right)$ & $h_{3}=\cosh x \cosh y-1$ \\
\hline$\omega=\cos y \mathrm{~d} x \wedge \mathrm{d} y$ & $\omega=\mathrm{d} x \wedge \mathrm{d} y$ & $\omega=\cosh y \mathrm{~d} x \wedge \mathrm{d} y$ \\
\hline $\begin{array}{l}\text { - Oscillating NH space } \mathbf{N H}_{+}^{1+1} \\
\text { (Co-Euclidean space) }\end{array}$ & - Galilean plane $\mathbf{G}^{1+1}$ & $\begin{array}{l}\text { - Expanding NH space } \mathbf{N H}_{-}^{1+1} \\
\text { (Co-Minkowskian space) }\end{array}$ \\
\hline $\mathbf{S}_{[+], 0}^{2}=\operatorname{ISO}(2) / \mathbb{R}$ & $\mathbf{S}_{[0], 0}^{2}=\operatorname{IISO}(1) / \mathbb{R}$ & $\mathbf{S}_{[-], 0}^{2}=\operatorname{ISO}(1,1) / \mathbb{R}$ \\
\hline $\begin{array}{l}\mathrm{d} s^{2}=\mathrm{d} x^{2}, \mathrm{~d} s^{\prime 2}=\mathrm{d} y^{2} \text { on } x=\text { cte } \\
x \in(-\pi, \pi], y \in \mathbb{R}\end{array}$ & $\begin{array}{l}\mathrm{d} s^{2}=\mathrm{d} x^{2}, \mathrm{~d} s^{\prime 2}=\mathrm{d} y^{2} \text { on } x=\text { cte } \\
x \in \mathbb{R}, y \in \mathbb{R}\end{array}$ & $\begin{array}{l}\mathrm{d} s^{2}=\mathrm{d} x^{2}, \mathrm{~d}{s^{\prime 2}}^{2}=\mathrm{d} y^{2} \text { on } x=\text { cte } \\
x \in \mathbb{R}, y \in \mathbb{R}\end{array}$ \\
\hline$V \simeq \mathfrak{i s o}(2) \simeq \mathfrak{s o}(2) \ltimes \mathbb{R}^{2}$ & $V \simeq \mathfrak{i i s o}(1) \simeq \mathbb{R} \ltimes \mathbb{R}^{2}$ & $V \simeq \mathfrak{i s o}(1,1) \simeq \mathfrak{s o}(1,1) \ltimes \mathbb{R}^{2}$ \\
\hline$X_{1}=\partial_{x}$ & $X_{1}=\partial_{x}$ & $X_{1}=\partial_{x}$ \\
\hline$X_{2}=\cos x \partial_{y}$ & $X_{2}=\partial_{y}$ & $X_{2}=\cosh x \partial_{y}$ \\
\hline$X_{3}=-\sin x \partial_{y}$ & $X_{3}=-x \partial_{y}$ & $X_{3}=-\sinh x \partial_{y}$ \\
\hline $\mathcal{H}_{\omega} \simeq \overline{\mathfrak{i} \mathfrak{s}}(2)=\overline{\mathfrak{s o}(2) \ltimes \mathbb{R}^{2}}$ & $\mathcal{H}_{\omega} \simeq \overline{\mathfrak{i n s o}}(1)=\overline{\mathbb{R} \ltimes \mathbb{R}^{2}}$ & $\mathcal{H}_{\omega} \simeq \overline{\mathfrak{i} \mathfrak{s o}}(1,1)=\overline{\mathfrak{s o}(1,1) \ltimes \mathbb{R}^{2}}$ \\
\hline$h_{1}=y$ & $h_{1}=y$ & $h_{1}=y$ \\
\hline$h_{2}=-\sin x$ & $h_{2}=-x$ & $h_{2}=-\sinh x$ \\
\hline$h_{3}=1-\cos x$ & $h_{3}=\frac{1}{2} x^{2}$ & $h_{3}=\cosh x-1$ \\
\hline$\omega=\mathrm{d} x \wedge \mathrm{d} y$ & $\omega=\mathrm{d} x \wedge \mathrm{d} y$ & $\omega=\mathrm{d} x \wedge \mathrm{d} y$ \\
\hline $\begin{array}{l}\text { - Anti-de Sitter space } \mathbf{A d} \mathbf{S}^{1+1} \\
\text { (Co-hyperbolic space) }\end{array}$ & - Minkowskian plane $\mathbf{M}^{1+1}$ & $\begin{array}{l}\text { - De Sitter space } \mathbf{d} \mathbf{S}^{1+1} \\
\text { (Doubly hyperbolic space) }\end{array}$ \\
\hline $\mathbf{S}_{[+],-}^{2}=\mathrm{SO}(2,1) / \mathrm{SO}(1,1)$ & $\mathbf{S}_{[0],-}^{2}=\operatorname{ISO}(1,1) / \mathrm{SO}(1,1)$ & $\mathbf{S}_{[-],-}^{2}=\mathrm{SO}(2,1) / \mathrm{SO}(1,1)$ \\
\hline $\mathrm{d} s^{2}=\cosh ^{2} y \mathrm{~d} x^{2}-\mathrm{d} y^{2}$ & $\mathrm{~d} s^{2}=\mathrm{d} x^{2}-\mathrm{d} y^{2}$ & $\mathrm{~d} s^{2}=\cos ^{2} y \mathrm{~d} x^{2}-\mathrm{d} y^{2}$ \\
\hline$x \in(-\pi, \pi], y \in \mathbb{R}$ & $x \in \mathbb{R}, y \in \mathbb{R}$ & $x \in \mathbb{R}, y \in(-\pi, \pi]$ \\
\hline$V \simeq \mathfrak{s o}(2,1)$ & $V \simeq \mathfrak{i s o}(1,1) \simeq \mathfrak{s o}(1,1) \ltimes \mathbb{R}^{2}$ & $V \simeq \mathfrak{s o}(2,1)$ \\
\hline$X_{1}=\partial_{x}$ & $X_{1}=\partial_{x}$ & $X_{1}=\partial_{x}$ \\
\hline$X_{2}=-\sin x \tanh y \partial_{x}+\cos x \partial_{y}$ & $X_{2}=\partial_{y}$ & $X_{2}=\sinh x \tan y \partial_{x}+\cosh x \partial_{y}$ \\
\hline$X_{3}=-\cos x \tanh y \partial_{x}-\sin x \partial_{y}$ & $X_{3}=-y \partial_{x}-x \partial_{y}$ & $X_{3}=-\cosh x \tan y \partial_{x}-\sinh x \partial_{y}$ \\
\hline $\mathcal{H}_{\omega} \simeq \overline{\mathfrak{s o}}(2,1) \simeq \mathfrak{s o}(2,1) \oplus \mathbb{R}$ & $\mathcal{H}_{\omega} \simeq \overline{\mathfrak{i} \mathfrak{s o}}(1,1)=\overline{\mathfrak{s o}(1,1) \ltimes \mathbb{R}^{2}}$ & $\mathcal{H}_{\omega} \simeq \overline{\mathfrak{s o}}(2,1) \simeq \overline{\mathfrak{s o}(2,1) \oplus \mathbb{R}}$ \\
\hline$h_{1}=\sinh y$ & $h_{1}=y$ & $h_{1}=\sin y$ \\
\hline$h_{2}=-\sin x \cosh y$ & $h_{2}=-x$ & $h_{2}=-\sinh x \cos y$ \\
\hline$h_{3}=1-\cos x \cosh y$ & $h_{3}=\frac{1}{2}\left(x^{2}-y^{2}\right)$ & $h_{3}=\cosh x \cos y-1$ \\
\hline$\omega=\cosh y \mathrm{~d} x \wedge \mathrm{d} y$ & $\omega=\mathrm{d} x \wedge \mathrm{d} y$ & $\omega=\cos y \mathrm{~d} x \wedge \mathrm{d} y$ \\
\hline
\end{tabular}


$(a=0,1,2,3)$ where $h_{\kappa, a}$ are now the Hamiltonians functions (4.6). This gives rise to two $t$-independent constants of motion for the system $X_{\kappa}$ (4.2) of the form

$$
F_{\kappa}:=D\left(C_{\kappa}\right), \quad F_{\kappa}^{(2)}:=D^{(2)}\left(\Delta\left(C_{\kappa}\right)\right) .
$$

The former turns out to be trivial, $F_{\kappa}=0$, meanwhile the latter can be written as

$$
\begin{aligned}
F_{\kappa}^{(2)} & =\frac{1}{\kappa_{1}}\left(1-\mathrm{C}_{\kappa_{1}}\left(x_{1}-x_{2}\right) \mathrm{C}_{\kappa_{1} \kappa_{2}}\left(y_{1}\right) \mathrm{C}_{\kappa_{1} \kappa_{2}}\left(y_{2}\right)-\kappa_{1} \kappa_{2} \mathrm{~S}_{\kappa_{1} \kappa_{2}}\left(y_{1}\right) \mathrm{S}_{\kappa_{1} \kappa_{2}}\left(y_{2}\right)\right) \\
& =\mathrm{V}_{\kappa_{1}}\left(x_{1}-x_{2}\right) \mathrm{C}_{\kappa_{1} \kappa_{2}}\left(y_{1}\right) \mathrm{C}_{\kappa_{1} \kappa_{2}}\left(y_{2}\right)+\kappa_{2} \mathrm{~V}_{\kappa_{1} \kappa_{2}}\left(y_{1}-y_{2}\right)
\end{aligned}
$$

where we have used the relations

$$
\mathrm{C}_{\kappa}(u \pm v)=\mathrm{C}_{\kappa}(u) \mathrm{C}_{\kappa}(v) \mp \kappa \mathrm{S}_{\kappa}(u) \mathrm{S}_{\kappa}(v), \quad \mathrm{S}_{\kappa}(u \pm v)=\mathrm{S}_{\kappa}(u) \mathrm{C}_{\kappa}(v) \pm \mathrm{C}_{\kappa}(u) \mathrm{S}_{\kappa}(v) .
$$

Note that the second expression for $F_{\kappa}^{(2)}$ admits the direct flat contraction $\kappa_{1}=0$; explicitly, since $\mathrm{V}_{0}(u)=u^{2} / 2$, then

$$
F_{\kappa_{1}=0, \kappa_{2}}^{(2)}=\frac{1}{2}\left[\left(x_{1}-x_{2}\right)^{2}+\kappa_{2}\left(y_{1}-y_{2}\right)^{2}\right],
$$

so that for $\kappa_{2}=+1$ we recover the Euclidean constant of motion (2.11).

We stress that, in fact, this constant of motion corresponds to the geodesic distance $s_{1}$ between two points $\left(x_{1}, y_{1}\right)$ and $\left(x_{2}, y_{2}\right)$ on the space $\mathbf{S}_{\left[\kappa_{1}\right], \kappa_{2}}^{2}$, which is given by [28]

$$
\mathrm{C}_{\kappa_{1}}\left(s_{1}\right)=\mathrm{C}_{\kappa_{1}}\left(x_{1}-x_{2}\right) \mathrm{C}_{\kappa_{1} \kappa_{2}}\left(y_{1}\right) \mathrm{C}_{\kappa_{1} \kappa_{2}}\left(y_{2}\right)+\kappa_{1} \kappa_{2} \mathrm{~S}_{\kappa_{1} \kappa_{2}}\left(y_{1}\right) \mathrm{S}_{\kappa_{1} \kappa_{2}}\left(y_{2}\right)
$$

Recall that $F_{\kappa}^{(2)}$ is a $t$-independent constant of motion for the diagonal prolongation $\widetilde{X}_{\kappa}$ of $X_{\kappa}$ to the manifold $\mathbf{S}_{\left[\kappa_{1}\right], \kappa_{2}}^{2} \times \mathbf{S}_{\left[\kappa_{1}\right], \kappa_{2}}^{2}$ (cf. [2]); namely, if $X_{\kappa}=X(x, y) \partial_{x}+Y(x, y) \partial_{y}$, then

$$
\tilde{X}_{\kappa}=X\left(x_{1}, y_{1}\right) \frac{\partial}{\partial x_{1}}+Y\left(x_{1}, y_{1}\right) \frac{\partial}{\partial y_{1}}+X\left(x_{2}, y_{2}\right) \frac{\partial}{\partial x_{2}}+Y\left(x_{2}, y_{2}\right) \frac{\partial}{\partial y_{2}}
$$

where $\left(\left(x_{1}, y_{1}\right),\left(x_{2}, y_{2}\right)\right) \in \mathbf{S}_{\left[\kappa_{1}\right], \kappa_{2}}^{2} \times \mathbf{S}_{\left[\kappa_{1}\right], \kappa_{2}}^{2}$. Moreover, the function $F_{\kappa}^{(2)}$ gives rise to two other constants of motion through the permutation $S_{i j}$ of the variables $\left(x_{i}, y_{i}\right) \leftrightarrow\left(x_{j}, y_{j}\right)$; these are

$$
F_{\kappa, 13}^{(2)}=S_{13}\left(F_{\kappa}^{(2)}\right), \quad F_{\kappa, 23}^{(2)}=S_{23}\left(F_{\kappa}^{(2)}\right) .
$$

Since prolongations are invariant under permutations, the functions $F_{\kappa, i j}^{(2)}$ are also $t$-independent constants of motion for the diagonal prolongations $\widetilde{X}_{\kappa}$ to $\mathbf{S}_{\left[\kappa_{1}\right], \kappa_{2}}^{2} \times \mathbf{S}_{\left[\kappa_{1}\right], \kappa_{2}}^{2}$.

By taking into account the expressions (5.2), (5.4), and (5.5), we can write the above three constants of motion in the form

$$
\begin{aligned}
F_{\kappa}^{(2)} & =\frac{1}{\kappa_{1}}\left(1-\mathrm{C}_{\kappa_{1}}\left(x_{1}-x_{2}\right) \mathrm{C}_{\kappa_{1} \kappa_{2}}\left(y_{1}\right) \mathrm{C}_{\kappa_{1} \kappa_{2}}\left(y_{2}\right)-\kappa_{1} \kappa_{2} \mathrm{~S}_{\kappa_{1} \kappa_{2}}\left(y_{1}\right) \mathrm{S}_{\kappa_{1} \kappa_{2}}\left(y_{2}\right)\right) \\
& =\frac{1}{\kappa_{1}}\left(1-\mathrm{C}_{\kappa_{1}}\left(s_{1}\right)\right)=\mathrm{V}_{\kappa_{1}}\left(s_{1}\right), \\
F_{\kappa, 23}^{(2)} & =\frac{1}{\kappa_{1}}\left(1-\mathrm{C}_{\kappa_{1}}\left(x_{1}-x_{3}\right) \mathrm{C}_{\kappa_{1} \kappa_{2}}\left(y_{1}\right) \mathrm{C}_{\kappa_{1} \kappa_{2}}\left(y_{3}\right)-\kappa_{1} \kappa_{2} \mathrm{~S}_{\kappa_{1} \kappa_{2}}\left(y_{1}\right) \mathrm{S}_{\kappa_{1} \kappa_{2}}\left(y_{3}\right)\right) \\
& =\frac{1}{\kappa_{1}}\left(1-\mathrm{C}_{\kappa_{1}}\left(s_{2}\right)\right)=\mathrm{V}_{\kappa_{1}}\left(s_{2}\right), \\
F_{\kappa, 13}^{(2)} & =\frac{1}{\kappa_{1}}\left(1-\mathrm{C}_{\kappa_{1}}\left(x_{3}-x_{2}\right) \mathrm{C}_{\kappa_{1} \kappa_{2}}\left(y_{3}\right) \mathrm{C}_{\kappa_{1} \kappa_{2}}\left(y_{2}\right)-\kappa_{1} \kappa_{2} \mathrm{~S}_{\kappa_{1} \kappa_{2}}\left(y_{3}\right) \mathrm{S}_{\kappa_{1} \kappa_{2}}\left(y_{2}\right)\right) \\
& =\frac{1}{\kappa_{1}}\left(1-\mathrm{C}_{\kappa_{1}}\left(s_{3}\right)\right)=\mathrm{V}_{\kappa_{1}}\left(s_{3}\right),
\end{aligned}
$$

where $s_{1}, s_{2}, s_{3}$ are three positive real constants. 


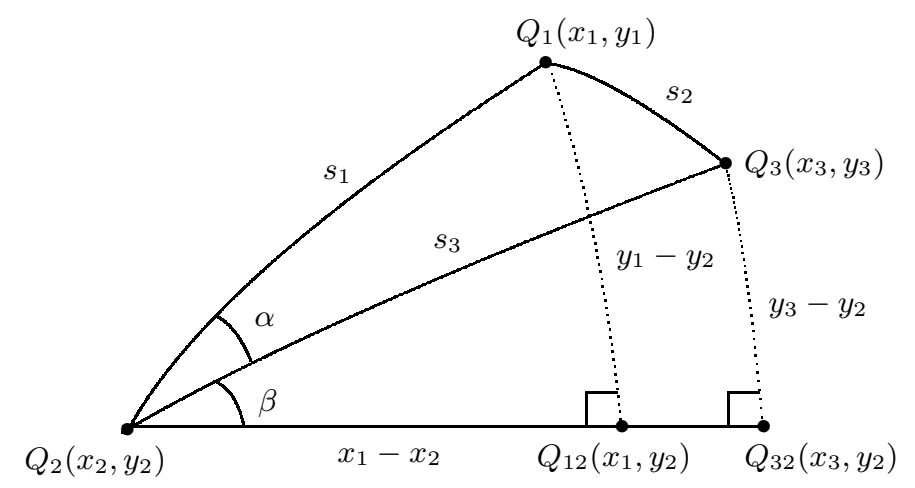

Figure 2: Triangles and geodesic distances involved in the derivation of the superposition rules for the LH system $X_{\kappa}$ (4.2) on the CK space $\mathbf{S}_{\left[\kappa_{1}\right], \kappa_{2}}^{2}$ (3.7).

\subsection{Superposition rules}

Since $\partial\left(F_{\kappa}^{(2)}, F_{\kappa, 23}^{(2)}\right) / \partial\left(x_{1}, y_{1}\right) \neq 0$, both constants of motion (5.6) are functionally independent functions. This, in turn, means that one can express the general solution $\left(x_{1}(t), y_{1}(t)\right)$ of the LH system $X_{\kappa}$ (4.2) in terms of two different particular solutions $\left(x_{2}(t), y_{2}(t)\right),\left(x_{3}(t), y_{3}(t)\right)$, and the two constants $s_{1}, s_{2}$. Therefore, one may start with such explicit expressions (5.6) and try to compute the superposition rules. Nevertheless, such a 'direct' procedure is frequently cumbersome and non-trivial. By contrast, we shall be able to obtain a closed analytical form for the superposition rules by applying a geometric approach based on the trigonometry of the CK spaces $\mathbf{S}_{\left[\kappa_{1}\right], \kappa_{2}}^{2}$ (3.7). All the trigonometric relations as well as generalized theorems used in our procedure can be found in $[29$.

With this aim, we set the points $Q_{1}:=\left(x_{1}, y_{1}\right), Q_{2}:=\left(x_{2}, y_{2}\right)$, and $Q_{3}:=\left(x_{3}, y_{3}\right)$ in $\mathbf{S}_{\left[\kappa_{1}\right], \kappa_{2}}^{2}$ forming a triangle $\triangle Q_{1} Q_{2} Q_{3}$. Its sides are geodesics such that the positive constants $s_{1}, s_{2}$ and $s_{3}$ appearing in (5.6) are, in this order, the geodesic distances $\overline{Q_{1} Q_{2}}, \overline{Q_{1} Q_{3}}$ and $\overline{Q_{3} Q_{2}}$, so fulfilling (5.4), and $\alpha$ is the angle between the geodesics $Q_{1} Q_{2}$ and $Q_{3} Q_{2}$; this is depicted in figure 2

Consider the orthogonal triangle $\triangle Q_{1} Q_{2} Q_{12}$ where $Q_{12}=\left(x_{1}, y_{2}\right)$, such that the geodesics $Q_{12} Q_{2}$ and $Q_{1} Q_{12}$ are orthogonal at $Q_{12}$ and with inner angle $\alpha+\beta$ at $Q_{2}$, as shown in figure 2 The cosine and sine theorems for this triangle, with geodesic distances $\overline{Q_{1} Q_{12}}=y_{1}-y_{2}>0$ and $\overline{Q_{12} Q_{2}}=x_{1}-x_{2}>0$, read

$$
\mathrm{C}_{\kappa_{1}}\left(s_{1}\right)=\mathrm{C}_{\kappa_{1}}\left(x_{1}-x_{2}\right) \mathrm{C}_{\kappa_{1} \kappa_{2}}\left(y_{1}-y_{2}\right), \quad \mathrm{S}_{\kappa_{1} \kappa_{2}}\left(y_{1}-y_{2}\right)=\mathrm{S}_{\kappa_{1}}\left(s_{1}\right) \mathrm{S}_{\kappa_{2}}(\alpha+\beta) .
$$

From these, we find that

$$
\mathrm{T}_{\kappa_{1}}\left(x_{1}-x_{2}\right)=\mathrm{T}_{\kappa_{1}}\left(s_{1}\right) \mathrm{C}_{\kappa_{2}}(\alpha+\beta) .
$$

After using the formulas (5.3), we arrive at

$$
\begin{aligned}
& \mathrm{T}_{\kappa_{1}}\left(x_{1}-x_{2}\right)=\mathrm{T}_{\kappa_{1}}\left(s_{1}\right)\left[\mathrm{C}_{\kappa_{2}}(\alpha) \mathrm{C}_{\kappa_{2}}(\beta)-\kappa_{2} \mathrm{~S}_{\kappa_{2}}(\alpha) \mathrm{S}_{\kappa_{2}}(\beta)\right], \\
& \mathrm{S}_{\kappa_{1} \kappa_{2}}\left(y_{1}-y_{2}\right)=\mathrm{S}_{\kappa_{1}}\left(s_{1}\right)\left[\mathrm{S}_{\kappa_{2}}(\alpha) \mathrm{C}_{\kappa_{2}}(\beta)+\mathrm{S}_{\kappa_{2}}(\beta) \mathrm{C}_{\kappa_{2}}(\alpha)\right] .
\end{aligned}
$$

Therefore, we need to express $\mathrm{C}_{\kappa_{2}}(\alpha), \mathrm{S}_{\kappa_{2}}(\alpha), \mathrm{C}_{\kappa_{2}}(\beta)$ and $\mathrm{S}_{\kappa_{2}}(\beta)$ in terms of $\left(x_{2}(t), y_{2}(t)\right),\left(x_{3}(t), y_{3}(t)\right)$ and the positive constants $s_{1}, s_{2}, s_{3}$.

Firstly, as above, if we now take the orthogonal triangle $\triangle Q_{2} Q_{3} Q_{32}$ where $Q_{32}=\left(x_{3}, y_{2}\right)$ with the geodesics $Q_{32} Q_{2}$ and $Q_{3} Q_{32}$ being orthogonal at $Q_{32}$ and with inner angle $\beta$ at $Q_{2}$, we can write

$$
\mathrm{C}_{\kappa_{2}}(\beta)=\frac{\mathrm{T}_{\kappa_{1}}\left(x_{3}-x_{2}\right)}{\mathrm{T}_{\kappa_{1}}\left(s_{3}\right)}, \quad \mathrm{S}_{\kappa_{2}}(\beta)=\frac{\mathrm{S}_{\kappa_{1} \kappa_{2}}\left(y_{3}-y_{2}\right)}{\mathrm{S}_{\kappa_{1}}\left(s_{3}\right)},
$$

where we have made use of the geodesic distances $\overline{Q_{32} Q_{2}}=x_{3}-x_{2}>0$ and $\overline{Q_{3} Q_{32}}=y_{3}-y_{2}>0$.

Secondly, in order to get $\mathrm{C}_{\kappa_{2}}(\alpha)$ and $\mathrm{S}_{\kappa_{2}}(\alpha)$, we consider the 'initial' triangle $\triangle Q_{1} Q_{2} Q_{3}$. By one hand, the cosine theorem gives

$$
\mathrm{C}_{\kappa_{2}}(\alpha)=\frac{\mathrm{C}_{\kappa_{1}}\left(s_{2}\right)-\mathrm{C}_{\kappa_{1}}\left(s_{1}\right) \mathrm{C}_{\kappa_{1}}\left(s_{3}\right)}{\kappa_{1} \mathrm{~S}_{\kappa_{1}}\left(s_{1}\right) \mathrm{S}_{\kappa_{1}}\left(s_{3}\right)} .
$$


On the other hand, $\mathrm{S}_{\kappa_{2}}(\alpha)$ can be written in terms of the area $A$ of this triangle through the generalized Cagnoli's theorem

$$
\mathrm{S}_{\kappa_{2}}(\alpha)=\frac{4 \mathrm{C}_{\kappa_{1}}\left(\frac{s_{1}}{2}\right) \mathrm{C}_{\kappa_{1}}\left(\frac{s_{2}}{2}\right) \mathrm{C}_{\kappa_{1}}\left(\frac{s_{3}}{2}\right) \mathrm{S}_{\kappa_{1}^{2} \kappa_{2}}\left(\frac{A}{2}\right)}{\mathrm{S}_{\kappa_{1}}\left(s_{1}\right) \mathrm{S}_{\kappa_{1}}\left(s_{3}\right)}
$$

Consequently, by substituting (5.8), (5.9) and (5.10) in (5.7), we obtain that

$$
\begin{aligned}
\mathrm{T}_{\kappa_{1}}\left(x_{1}-x_{2}\right)= & \mathrm{T}_{\kappa_{1}}\left(x_{3}-x_{2}\right) \frac{\mathrm{C}_{\kappa_{1}}\left(s_{2}\right)-\mathrm{C}_{\kappa_{1}}\left(s_{1}\right) \mathrm{C}_{\kappa_{1}}\left(s_{3}\right)}{\kappa_{1} \mathrm{C}_{\kappa_{1}}\left(s_{1}\right) \mathrm{S}_{\kappa_{1}}\left(s_{3}\right) \mathrm{T}_{\kappa_{1}}\left(s_{3}\right)} \\
& -4 \kappa_{2} \mathrm{~S}_{\kappa_{1} \kappa_{2}}\left(y_{3}-y_{2}\right) \frac{\mathrm{C}_{\kappa_{1}}\left(\frac{s_{1}}{2}\right) \mathrm{C}_{\kappa_{1}}\left(\frac{s_{2}}{2}\right) \mathrm{C}_{\kappa_{1}}\left(\frac{s_{3}}{2}\right) \mathrm{S}_{\kappa_{1}^{2} \kappa_{2}}\left(\frac{A}{2}\right)}{\mathrm{C}_{\kappa_{1}}\left(s_{1}\right) \mathrm{S}_{\kappa_{1}}^{2}\left(s_{3}\right)}, \\
\mathrm{S}_{\kappa_{1} \kappa_{2}}\left(y_{1}-y_{2}\right)= & \mathrm{S}_{\kappa_{1} \kappa_{2}}\left(y_{3}-y_{2}\right) \frac{\mathrm{C}_{\kappa_{1}}\left(s_{2}\right)-\mathrm{C}_{\kappa_{1}}\left(s_{1}\right) \mathrm{C}_{\kappa_{1}}\left(s_{3}\right)}{\kappa_{1} \mathrm{~S}_{\kappa_{1}}^{2}\left(s_{3}\right)} \\
& +4 \mathrm{~T}_{\kappa_{1}}\left(x_{3}-x_{2}\right) \frac{\mathrm{C}_{\kappa_{1}}\left(\frac{s_{1}}{2}\right) \mathrm{C}_{\kappa_{1}}\left(\frac{s_{2}}{2}\right) \mathrm{C}_{\kappa_{1}}\left(\frac{s_{3}}{2}\right) \mathrm{S}_{\kappa_{1}^{2} \kappa_{2}}\left(\frac{A}{2}\right)}{\mathrm{S}_{\kappa_{1}}\left(s_{3}\right) \mathrm{T}_{\kappa_{1}}\left(s_{3}\right)} .
\end{aligned}
$$

These relations can further be written in different ways by considering the expressions for the area $A$ presented in 29]. For instance, for the six spaces with $\kappa_{2} \neq 0$ (so precluding the three Newtonian spaces), there exists a generalized L'Huillier formula $A=A\left(s_{1}, s_{2}, s_{3}\right)$, which is the curved counterpart of the Heron-Archimedes area formula (2.14) for the Euclidean plane with $\left(\kappa_{1}, \kappa_{2}\right)=(0,+1)$, given by

$$
\begin{aligned}
& \mathrm{T}_{\kappa_{1}^{2} \kappa_{2}}^{2}\left(\frac{A}{4}\right)=\frac{1}{\kappa_{2}} \mathrm{~T}_{\kappa_{1}}\left(\frac{p}{2}\right) \mathrm{T}_{\kappa_{1}}\left(\frac{p-s_{1}}{2}\right) \mathrm{T}_{\kappa_{1}}\left(\frac{p-s_{2}}{2}\right) \mathrm{T}_{\kappa_{1}}\left(\frac{p-s_{3}}{2}\right), \quad \kappa_{2} \neq 0, \\
& p=\frac{1}{2}\left(s_{1}+s_{2}+s_{3}\right),
\end{aligned}
$$

such that $p$ is one-half the sum of the three geodesic sides of the triangle $\triangle Q_{1} Q_{2} Q_{3}$. In particular, for the flat Euclidean $\left(\kappa_{2}>0\right)$ and Minkowskian $\left(\kappa_{2}<0\right)$ spaces, this expression reduces to

$$
A^{2}=\frac{1}{\kappa_{2}} p\left(p-s_{1}\right)\left(p-s_{2}\right)\left(p-s_{3}\right)=\frac{1}{16 \kappa_{2}}\left[2\left(s_{1}^{2} s_{2}^{2}+s_{1}^{2} s_{3}^{2}+s_{2}^{2} s_{3}^{2}\right)-\left(s_{1}^{4}+s_{2}^{4}+s_{3}^{4}\right)\right],
$$

which is just (2.14) for $\kappa_{2}=+1$ and $s_{i}:=k_{i}$.

We stress that there exists a second solution for the superposition rules (see the Euclidean case (2.14)), say $\left(x_{1}^{-}, y_{1}^{-}\right)$, which corresponds to change the sign of the last term in both relations (5.11). This can be proven in a similar way by considering another configuration for the triangles.

We summarize the results of this section in the following statement.

Theorem 1. Let $X_{\kappa}$ be the LH system (4.2) defined on the CK space $\mathbf{S}_{\left[\kappa_{1}\right], \kappa_{2}}^{2}$, with vector fields 4.1], Hamiltonian functions (4.6) and symplectic form 4.5). Then:

(i) The functions (5.6) are three t-independent constants of motion for the diagonal prolongation $\widetilde{X}_{\boldsymbol{\kappa}}$ to the manifold $\mathbf{S}_{\left[\kappa_{1}\right], \kappa_{2}}^{2} \times \mathbf{S}_{\left[\kappa_{1}\right], \kappa_{2}}^{2} \times \mathbf{S}_{\left[\kappa_{1}\right], \kappa_{2}}^{2}$, such that any pair among them is formed by two functionally independent functions.

(ii) The general solution $\left(x_{1}(t), y_{1}(t)\right)$ of $X_{\kappa}$ in terms of two different particular solutions $\left(x_{2}(t), y_{2}(t)\right)$ and $\left(x_{3}(t), y_{3}(t)\right)$ can be written as

$$
\begin{aligned}
\mathrm{T}_{\kappa_{1}}\left(x_{1}^{ \pm}-x_{2}\right)= & \mathrm{T}_{\kappa_{1}}\left(x_{3}-x_{2}\right) \frac{\mathrm{C}_{\kappa_{1}}\left(s_{2}\right)-\mathrm{C}_{\kappa_{1}}\left(s_{1}\right) \mathrm{C}_{\kappa_{1}}\left(s_{3}\right)}{\kappa_{1} \mathrm{C}_{\kappa_{1}}\left(s_{1}\right) \mathrm{S}_{\kappa_{1}}\left(s_{3}\right) \mathrm{T}_{\kappa_{1}}\left(s_{3}\right)} \\
& \mp 4 \kappa_{2} \mathrm{~S}_{\kappa_{1} \kappa_{2}}\left(y_{3}-y_{2}\right) \frac{\mathrm{C}_{\kappa_{1}}\left(\frac{s_{1}}{2}\right) \mathrm{C}_{\kappa_{1}}\left(\frac{s_{2}}{2}\right) \mathrm{C}_{\kappa_{1}}\left(\frac{s_{3}}{2}\right) \mathrm{S}_{\kappa_{1}^{2} \kappa_{2}}\left(\frac{A}{2}\right)}{\mathrm{C}_{\kappa_{1}}\left(s_{1}\right) \mathrm{S}_{\kappa_{1}}^{2}\left(s_{3}\right)} \\
\mathrm{S}_{\kappa_{1} \kappa_{2}}\left(y_{1}^{ \pm}-y_{2}\right)= & \mathrm{S}_{\kappa_{1} \kappa_{2}}\left(y_{3}-y_{2}\right) \frac{\mathrm{C}_{\kappa_{1}}\left(s_{2}\right)-\mathrm{C}_{\kappa_{1}}\left(s_{1}\right) \mathrm{C}_{\kappa_{1}}\left(s_{3}\right)}{\kappa_{1} \mathrm{~S}_{\kappa_{1}}^{2}\left(s_{3}\right)} \\
& \pm 4 \mathrm{~T}_{\kappa_{1}}\left(x_{3}-x_{2}\right) \frac{\mathrm{C}_{\kappa_{1}}\left(\frac{s_{1}}{2}\right) \mathrm{C}_{\kappa_{1}}\left(\frac{s_{2}}{2}\right) \mathrm{C}_{\kappa_{1}}\left(\frac{s_{3}}{2}\right) \mathrm{S}_{\kappa_{1}^{2} \kappa_{2}}\left(\frac{A}{2}\right)}{\mathrm{S}_{\kappa_{1}}\left(s_{3}\right) \mathrm{T}_{\kappa_{1}}\left(s_{3}\right)}
\end{aligned}
$$


where $s_{1}, s_{2}, s_{3}$, and $A$ are positive constants, such that the latter is just the area of triangle formed by the three solutions considered as points in $\mathbf{S}_{\left[\kappa_{1}\right], \kappa_{2}}^{2}$, meanwhile the former ones are its geodesic sides.

\subsection{Discussion}

We illustrate the above results by writing the Casimir (5.1), the constant of motion $F_{\kappa}^{(2)}$ (5.6), and the superposition rules (5.12) in table 2 for each of the nine CK spaces $\mathbf{S}_{\left[\kappa_{1}\right], \kappa_{2}}^{2}$ (3.7) with the same structure of table 1. We remark that tables 1 and 2 comprise the main results of the paper. When both tables are read by rows, one finds, in this order, the three classical Riemannian spaces of constant curvature $\kappa_{1}$ with $\kappa_{2}>0$, the semi-Riemannian spaces or Newtonian spacetimes of constant curvature $\kappa_{1}$ with $\kappa_{2}=0$ $(c \rightarrow \infty)$, and the pseudo-Riemannian spaces or Lorentzian spacetimes of constant curvature $\kappa_{1}=-\Lambda$ with $\kappa_{2}=-1 / c^{2}<0$. When these are read by columns, one finds three spaces of positive, zero (flat) and negative constant curvature, correspondingly, but with different metric signature $\operatorname{diag}\left(+1, \kappa_{2}\right)$.

Clearly, all the Euclidean results previously obtained in [9], and here summarized in section 2, are recovered for $\mathbf{S}_{[0],+}^{2}$ such that the three constants $k_{i}(i=1,2,3)$ coincide with the geodesic distances $s_{i}$. We recall that for the three flat spaces with $\kappa_{1}=0$ (middle column of the tables), $\mathbf{S}_{[0], \kappa_{2}}^{2}$, the contraction of the constant of motion $F_{\kappa}^{(2)}$ comes out directly from the expression written in terms of $\kappa$-versed sines in (5.2). Similarly, the contraction of the superposition rules (5.12) can be obtained by taking into account that the factor

$$
\frac{\mathrm{C}_{\kappa_{1}}\left(s_{2}\right)-\mathrm{C}_{\kappa_{1}}\left(s_{1}\right) \mathrm{C}_{\kappa_{1}}\left(s_{3}\right)}{\kappa_{1}}=\mathrm{V}_{\kappa_{1}}\left(s_{1}\right)+\mathrm{V}_{\kappa_{1}}\left(s_{3}\right)-\mathrm{V}_{\kappa_{1}}\left(s_{2}\right)-\kappa_{1} \mathrm{~V}_{\kappa_{1}}\left(s_{1}\right) \mathrm{V}_{\kappa_{1}}\left(s_{3}\right) \mapsto \frac{1}{2}\left(s_{1}^{2}+s_{3}^{2}-s_{2}^{2}\right),
$$

when $\kappa_{1}=0$, so avoiding to take power series in the curvature.

An important fact concerns the three semi-Riemannian or Newtonian spaces with $\kappa_{2}=0$ (middle row of the tables), $\mathbf{S}_{\left[\kappa_{1}\right], 0}^{2}$. The constants of motion (5.6) only includes the variables $x_{i}(i=1,2,3)$, that is,

$$
F_{\kappa_{1}, \kappa_{2}=0}^{(2)}=\frac{1}{\kappa_{1}}\left(1-\mathrm{C}_{\kappa_{1}}\left(x_{1}-x_{2}\right)\right)=\frac{1}{\kappa_{1}}\left(1-\mathrm{C}_{\kappa_{1}}\left(s_{1}\right)\right) .
$$

Therefore, strictly speaking, only a part of their superposition rules can be derived from them, which corresponds to the first relation of (5.12):

$$
\mathrm{T}_{\kappa_{1}}\left(x_{1}^{ \pm}-x_{2}\right)=\mathrm{T}_{\kappa_{1}}\left(x_{3}-x_{2}\right) \frac{\mathrm{C}_{\kappa_{1}}\left(s_{2}\right)-\mathrm{C}_{\kappa_{1}}\left(s_{1}\right) \mathrm{C}_{\kappa_{1}}\left(s_{3}\right)}{\kappa_{1} \mathrm{C}_{\kappa_{1}}\left(s_{1}\right) \mathrm{S}_{\kappa_{1}}\left(s_{3}\right) \mathrm{T}_{\kappa_{1}}\left(s_{3}\right)} .
$$

Nevertheless, we stress that the complete superposition rules (5.12) also apply for these spaces in such a manner that the 'missing' part, containing the variables $y_{i}$, is consistently obtained through the contraction procedure. This corresponds to the second expression in (5.12), namely

$$
y_{1}^{ \pm}-y_{2}=\left(y_{3}-y_{2}\right) \frac{\mathrm{C}_{\kappa_{1}}\left(s_{2}\right)-\mathrm{C}_{\kappa_{1}}\left(s_{1}\right) \mathrm{C}_{\kappa_{1}}\left(s_{3}\right)}{\kappa_{1} \mathrm{~S}_{\kappa_{1}}^{2}\left(s_{3}\right)} \pm 2 \mathrm{~T}_{\kappa_{1}}\left(x_{3}-x_{2}\right) \frac{\mathrm{C}_{\kappa_{1}}\left(\frac{s_{1}}{2}\right) \mathrm{C}_{\kappa_{1}}\left(\frac{s_{2}}{2}\right) \mathrm{C}_{\kappa_{1}}\left(\frac{s_{3}}{2}\right) A}{\mathrm{~S}_{\kappa_{1}}\left(s_{3}\right) \mathrm{T}_{\kappa_{1}}\left(s_{3}\right)} .
$$

Let us explain this point from a trigonometry procedure. Consider the triangles of figure 2 that represent the solution $\left(x_{1}^{+}, y_{1}^{+}\right)$of the superposition rules. Trigonometry on $\mathbf{S}_{\left[\kappa_{1}\right], 0}^{2}$ gives the relations

$$
s_{1}=x_{1}-x_{2}, \quad s_{2}=x_{3}-x_{1}, \quad s_{3}=x_{3}-x_{2}, \quad s_{3}=s_{1}+s_{2},
$$

so that

$$
\mathrm{S}_{\kappa_{1}}\left(s_{1}\right)=\frac{\mathrm{C}_{\kappa_{1}}\left(s_{2}\right)-\mathrm{C}_{\kappa_{1}}\left(s_{1}\right) \mathrm{C}_{\kappa_{1}}\left(s_{3}\right)}{\kappa_{1} \mathrm{~S}_{\kappa_{1}}\left(s_{3}\right)}, \quad \mathrm{T}_{\kappa_{1}}\left(s_{3}\right)=\mathrm{T}_{\kappa_{1}}\left(x_{3}-x_{2}\right) .
$$

The sine theorem on the orthogonal triangles $\triangle Q_{1} Q_{2} Q_{12}$ and $\triangle Q_{2} Q_{3} Q_{32}$ reads

$$
y_{1}-y_{2}=\mathrm{S}_{\kappa_{1}}\left(s_{1}\right)(\alpha+\beta), \quad y_{3}-y_{2}=\mathrm{S}_{\kappa_{1}}\left(s_{3}\right) \beta .
$$


Table 2: For each LH system $X_{\kappa}$ (4.2) on the CK space $\mathbf{S}_{\left[\kappa_{1}\right], \kappa_{2}}^{2}$ (3.7) it is displayed, according to the 'normalized' values of the contraction parameters $\kappa_{a} \in\{1,0,-1\}$, the Casimir $C_{\kappa}$ (5.1) , the constant of motion $F_{\kappa}^{(2)}$ (5.6) and the superposition rules (5.12) in geodesic parallel coordinates $(x, y)$ (3.10).

- Sphere $\mathbf{S}_{[+],+}^{2}=\mathbf{S}^{2}$

$C=v_{3} v_{0}-\frac{1}{2}\left(v_{1}^{2}+v_{2}^{2}+v_{3}^{2}\right)$

$F^{(2)}=1-\cos \left(x_{1}-x_{2}\right) \cos y_{1} \cos y_{2}-\sin y_{1} \sin y_{2}$

$=1-\cos s_{1}$

$\tan \left(x_{1}-x_{2}\right)=\tan \left(x_{3}-x_{2}\right) \frac{\cos s_{2}-\cos s_{1} \cos s_{3}}{\cos s_{1} \sin s_{3} \tan s_{3}}$

$\mp 4 \sin \left(y_{3}-y_{2}\right) \frac{\cos \left(\frac{s_{1}}{2}\right) \cos \left(\frac{s_{2}}{2}\right) \cos \left(\frac{s_{3}}{2}\right) \sin \left(\frac{A}{2}\right)}{\cos s_{1} \sin ^{2} s_{3}}$

$\sin \left(y_{1}-y_{2}\right)=\sin \left(y_{3}-y_{2}\right) \frac{\cos s_{2}-\cos s_{1} \cos s_{3}}{\sin ^{2} s_{3}}$

$\pm 4 \tan \left(x_{3}-x_{2}\right) \frac{\cos \left(\frac{s_{1}}{2}\right) \cos \left(\frac{s_{2}}{2}\right) \cos \left(\frac{s_{3}}{2}\right) \sin \left(\frac{A}{2}\right)}{\sin s_{3} \tan s_{3}}$

- Oscillating $\mathrm{NH}$ space $\mathbf{S}_{[+], 0}^{2}=\mathbf{N H}_{+}^{1+1}$

$C=v_{3} v_{0}-\frac{1}{2}\left(v_{2}^{2}+v_{3}^{2}\right)$

$F^{(2)}=1-\cos \left(x_{1}-x_{2}\right)=1-\cos s_{1}$

マ
- Euclidean plane $\mathbf{S}_{[0],+}^{2}=\mathbf{E}^{2}$

$C=v_{3} v_{0}-\frac{1}{2}\left(v_{1}^{2}+v_{2}^{2}\right)$

$$
F^{(2)}=\frac{1}{2}\left[\left(x_{1}-x_{2}\right)^{2}+\left(y_{1}-y_{2}\right)^{2}\right]
$$$$
=\frac{1}{2} s_{1}^{2}
$$

$x_{1}-x_{2}=\left(x_{3}-x_{2}\right) \frac{s_{1}^{2}+s_{3}^{2}-s_{2}^{2}}{2 s_{3}^{2}}$

$$
\mp 2\left(y_{3}-y_{2}\right) \frac{A}{s_{3}^{2}}
$$

$$
y_{1}-y_{2}=\left(y_{3}-y_{2}\right) \frac{s_{1}^{2}+s_{3}^{2}-s_{2}^{2}}{2 s_{3}^{2}}
$$

$$
\pm 2\left(x_{3}-x_{2}\right) \frac{A}{s_{3}^{2}}
$$

- Galilean plane $\mathbf{S}_{[0], 0}^{2}=\mathbf{G}^{1+1}$ $C=v_{3} v_{0}-\frac{1}{2} v_{2}^{2}$

$F^{(2)}=\frac{1}{2}\left(x_{1}-x_{2}\right)^{2}=\frac{1}{2} s_{1}^{2}$

$x_{1}-x_{2}=\left(x_{3}-x_{2}\right) \frac{s_{1}^{2}+s_{3}^{2}-s_{2}^{2}}{2 s_{3}^{2}}$

$y_{1}-y_{2}=\left(y_{3}-y_{2}\right) \frac{s_{1}^{2}+s_{3}^{2}-s_{2}^{2}}{2 s_{3}^{2}}$

$$
\pm 2\left(x_{3}-x_{2}\right) \frac{A}{s_{3}^{2}}
$$

- Minkowskian plane $\mathbf{S}_{[0],-}^{2}=\mathbf{M}^{1+1}$ $C=v_{3} v_{0}+\frac{1}{2}\left(v_{1}^{2}-v_{2}^{2}\right)$

$$
F^{(2)}=\frac{1}{2}\left[\left(x_{1}-x_{2}\right)^{2}-\left(y_{1}-y_{2}\right)^{2}\right]
$$

$=\frac{1}{2} s_{1}^{2}$

$x_{1}-x_{2}=\left(x_{3}-x_{2}\right) \frac{s_{1}^{2}+s_{3}^{2}-s_{2}^{2}}{2 s_{3}^{2}}$

$\tan \left(x_{1}-x_{2}\right)=\tan \left(x_{3}-x_{2}\right) \frac{\cos s_{2}-\cos s_{1} \cos s_{3}}{\cos s_{1} \sin s_{3} \tan s_{3}}$

$\pm 4 \sinh \left(y_{3}-y_{2}\right) \frac{\cos \left(\frac{s_{1}}{2}\right) \cos \left(\frac{s_{2}}{2}\right) \cos \left(\frac{s_{3}}{2}\right) \sinh \left(\frac{A}{2}\right)}{\cos s_{1} \sin ^{2} s_{3}}$

$\sinh \left(y_{1}-y_{2}\right)=\sinh \left(y_{3}-y_{2}\right) \frac{\cos s_{2}-\cos s_{1} \cos s_{3}}{\sin ^{2} s_{3}}$

$\pm 4 \tan \left(x_{3}-x_{2}\right) \frac{\cos \left(\frac{s_{1}}{2}\right) \cos \left(\frac{s_{2}}{2}\right) \cos \left(\frac{s_{3}}{2}\right) \sinh \left(\frac{A}{2}\right)}{\sin s_{3} \tan s_{3}}$

$y_{1}-y_{2}=\left(y_{3}-y_{2}\right) \frac{s_{1}^{2}+s_{3}^{2}-s_{2}^{2}}{2 s_{3}^{2}}$

$\pm 2\left(x_{3}-x_{2}\right) \frac{A}{s_{3}^{2}}$
- Hyperbolic space $\mathbf{S}_{[-],+}^{2}=\mathbf{H}^{2}$

$C=v_{3} v_{0}-\frac{1}{2}\left(v_{1}^{2}+v_{2}^{2}-v_{3}^{2}\right)$

$F^{(2)}=\cosh \left(x_{1}-x_{2}\right) \cosh y_{1} \cosh y_{2}-\sinh y_{1} \sinh y_{2}-1$

$=\cosh s_{1}-1$

$\tanh \left(x_{1}-x_{2}\right)=\tanh \left(x_{3}-x_{2}\right) \frac{\cosh s_{1} \cosh s_{3}-\cosh s_{2}}{\cosh s_{1} \sinh s_{3} \tanh s_{3}}$

$\mp 4 \sinh \left(y_{3}-y_{2}\right) \frac{\cosh \left(\frac{s_{1}}{2}\right) \cosh \left(\frac{s_{2}}{2}\right) \cosh \left(\frac{s_{3}}{2}\right) \sin \left(\frac{A}{2}\right)}{s_{1}}$

$\cosh s_{1} \sinh ^{2} s_{3}$

$\sinh \left(y_{1}-y_{2}\right)=\sinh \left(y_{3}-y_{2}\right) \frac{\cosh s_{1} \cosh s_{3}-\cosh s_{2}}{\sinh ^{2} s_{3}}$

$\pm 4 \tanh \left(x_{3}-x_{2}\right) \frac{\cosh \left(\frac{s_{1}}{2}\right) \cosh \left(\frac{s_{2}}{2}\right) \cosh \left(\frac{s_{3}}{2}\right) \sin \left(\frac{A}{2}\right)}{\sinh s_{3} \tanh s_{3}}$

- Expanding NH space $\mathbf{S}_{[-], 0}^{2}=\mathbf{N H}_{-}^{1+1}$

$C=v_{3} v_{0}-\frac{1}{2}\left(v_{2}^{2}-v_{3}^{2}\right)$

$F^{(2)}=\cosh \left(x_{1}-x_{2}\right)-1=\cosh s_{1}-1$

$\tanh \left(x_{1}-x_{2}\right)=\tanh \left(x_{3}-x_{2}\right) \frac{\cosh s_{1} \cosh s_{3}-\cosh s_{2}}{\cosh s_{1} \sinh s_{3} \tanh s_{3}}$

$y_{1}-y_{2}=\left(y_{3}-y_{2}\right) \frac{\cosh s_{1} \cosh s_{3}-\cosh s_{2}}{\sinh ^{2} s_{3}}$

$\pm 2 \tanh \left(x_{3}-x_{2}\right) \frac{\cosh \left(\frac{s_{1}}{2}\right) \cosh \left(\frac{s_{2}}{2}\right) \cosh \left(\frac{s_{3}}{2}\right) A}{\sinh s_{3} \tanh s_{3}}$

- De Sitter space $\mathbf{S}_{[-],-}^{2}=\mathbf{d} \mathbf{S}^{1+1}$

$C=v_{3} v_{0}+\frac{1}{2}\left(v_{1}^{2}-v_{2}^{2}+v_{3}^{2}\right)$

$F^{(2)}=\cosh \left(x_{1}-x_{2}\right) \cos y_{1} \cos y_{2}+\sin y_{1} \sin y_{2}-1$

$=\cosh s_{1}-1$

$\tanh \left(x_{1}-x_{2}\right)=\tanh \left(x_{3}-x_{2}\right) \frac{\cosh s_{1} \cosh s_{3}-\cosh s_{2}}{\cosh s_{1} \sinh s_{3} \tanh s_{3}}$

$\pm 4 \sin \left(y_{3}-y_{2}\right) \frac{\cosh \left(\frac{s_{1}}{2}\right) \cosh \left(\frac{s_{2}}{2}\right) \cosh \left(\frac{s_{3}}{2}\right) \sinh \left(\frac{A}{2}\right)}{\cosh s_{1} \sinh ^{2} s_{3}}$ $\sin \left(y_{1}-y_{2}\right)=\sin \left(y_{3}-y_{2}\right) \frac{\cosh s_{1} \cosh s_{3}-\cosh s_{2}}{\sinh ^{2} s_{3}}$

$\pm 4 \tanh \left(x_{3}-x_{2}\right) \frac{\cosh \left(\frac{s_{1}}{2}\right) \cosh \left(\frac{s_{2}}{2}\right) \cosh \left(\frac{s_{3}}{2}\right) \sinh \left(\frac{A}{2}\right)}{\sinh s_{3} \tanh s_{3}}$ 
The area of the triangle $\triangle Q_{1} Q_{2} Q_{3}$ is given by

$$
A=\frac{\mathrm{S}_{\kappa_{1}}\left(s_{1}\right) \mathrm{S}_{\kappa_{1}}\left(s_{3}\right)}{2 \mathrm{C}_{\kappa_{1}}\left(\frac{s_{1}}{2}\right) \mathrm{C}_{\kappa_{1}}\left(\frac{s_{2}}{2}\right) \mathrm{C}_{\kappa_{1}}\left(\frac{s_{3}}{2}\right)} \alpha
$$

Then, from (5.15) we find that

$$
y_{1}-y_{2}=\left(y_{3}-y_{2}\right) \frac{\mathrm{S}_{\kappa_{1}}\left(s_{1}\right)}{\mathrm{S}_{\kappa_{1}}\left(s_{3}\right)}+\mathrm{S}_{\kappa_{1}}\left(s_{1}\right) \alpha
$$

By substituting $\mathrm{S}_{\kappa_{1}}\left(s_{1}\right)$ (5.14) in the first term and $\alpha$ from (5.16) in the second one, we get

$$
y_{1}-y_{2}=\left(y_{3}-y_{2}\right) \frac{\mathrm{C}_{\kappa_{1}}\left(s_{2}\right)-\mathrm{C}_{\kappa_{1}}\left(s_{1}\right) \mathrm{C}_{\kappa_{1}}\left(s_{3}\right)}{\kappa_{1} \mathrm{~S}_{\kappa_{1}}^{2}\left(s_{3}\right)}+\frac{2 \mathrm{C}_{\kappa_{1}}\left(\frac{s_{1}}{2}\right) \mathrm{C}_{\kappa_{1}}\left(\frac{s_{2}}{2}\right) \mathrm{C}_{\kappa_{1}}\left(\frac{s_{3}}{2}\right)}{\mathrm{S}_{\kappa_{1}}\left(s_{3}\right)} A,
$$

which, by introducing $\mathrm{T}_{\kappa_{1}}\left(x_{3}-x_{2}\right)$ (5.14), leads to the solution $y_{1}^{+}$(5.13).

Finally, we would like to recall that for the Lorentzian spaces with $\kappa_{2}=-1 / c^{2}$ (third row of the tables), $\mathbf{S}_{\left[\kappa_{1}\right],-}^{2}$, the triangle $\triangle Q_{1} Q_{2} Q_{3}$ is a time-like one, that is, with elliptic distances $s_{i}$ for $\mathbf{A d} \mathbf{S}^{\mathbf{1 + 1}}$ $\left(\kappa_{1}>0\right)$ and hyperbolic ones for $\mathbf{d S}^{\mathbf{1 + 1}}\left(\kappa_{1}<0\right)$. Any orthogonal geodesic to a time-like one is space-like, so the distances $y_{1}-y_{2}$ and $y_{3}-y_{2}$ are hyperbolic in $\mathbf{A d \mathbf { d }} \mathbf{S}^{\mathbf{1 + 1}}$ and elliptic in $\mathbf{d} \mathbf{S}^{\mathbf{1 + 1}}$. In fact, both spaces are related through the interchange of time- and space-like geodesics. By contrast, in the Riemannian spaces with $\kappa_{2}>0$ (first row of the tables), $\mathbf{S}_{\left[\kappa_{1}\right],+}^{2}$, there is only one type of distance, elliptic for $\mathbf{S}^{2}$ and hyperbolic for $\mathbf{H}^{2}$.

\section{Concluding remarks}

We have achieved the first LH systems on 2D Riemannian, Lorentzian, and Newtonian spaces along with their constants of motion and superposition rules by following a geometrical CK approach. The graded contraction procedure based upon the two parameters $\left(\kappa_{1}, \kappa_{2}\right)$ (curvature and signature) provides a clear description of the relationships among all the structures involved, which have been explicitly shown in tables 1 and 2 for each specific space.

In this framework, some natural open problems arise, which could be expected to be solved by applying similar geometrical techniques, namely:

- All of these 2D LH systems could be extended to higher dimensions by starting from the known isometries on these spaces. The main point to be analyzed in this construction is the role that higher-order Casimirs play in relation with constants of motion. Recall that quasi-othogonal algebras, in any dimension, are always endowed with a second-order Casimir related to the KillingCartan form; in the 2D case this is the only one. Nevertheless, in three dimensions there is another third-order Casimir, in the 4D case there is a fourth-order one, etc. (see [38]).

- The class $\mathrm{P}_{2} \simeq \mathfrak{s l}(2) \simeq \mathfrak{s o}(2,1)$ of the classification of $2 \mathrm{D}$ Euclidean LH systems is spanned by the following vector fields in Cartesian coordinates $(x, y)$ [8, 9]:

$$
X_{1}:=\frac{\partial}{\partial x}, \quad X_{2}:=x \frac{\partial}{\partial x}+y \frac{\partial}{\partial y}, \quad X_{3}:=\left(x^{2}-y^{2}\right) \frac{\partial}{\partial x}+2 x y \frac{\partial}{\partial y} .
$$

Remarkably, these vector fields are conformal symmetries of the Euclidean plane $\mathbb{R}^{2}$. In particular, $X_{1}$ is the translation along the axis $x$, the vector field $X_{2}$ is a dilatation, and $X_{3}$ is a specific conformal transformation related to the $x$-axis. They also close on a Lie subalgebra of the conformal Euclidean algebra $\mathfrak{s o}(3,1)$. Moreover, such vector fields are also Hamiltonian vector fields relative to a symplectic form [8]. This suggests us to make use of the known conformal symmetries on the CK spaces 28] to develop the 'curved' counterparts of the LH systems of class $\mathrm{P}_{2}$. The physical relevance of this problem is due to the fact that $\mathrm{P}_{2}$-LH systems underly the complex Riccati equation and some Milne-Pinney and Kummer-Schwarz equations [9]. 
- The class $\mathrm{I}_{4} \simeq \mathfrak{s l}(2) \simeq \mathfrak{s o}(2,1)$ is spanned by the vector fields given by [8, 9 ]:

$$
X_{1}:=\frac{\partial}{\partial x}+\frac{\partial}{\partial y}, \quad X_{2}:=x \frac{\partial}{\partial x}+y \frac{\partial}{\partial y}, \quad X_{3}:=x^{2} \frac{\partial}{\partial x}+y^{2} \frac{\partial}{\partial y} .
$$

Each $X_{i}$ has also a clear interpretation as conformal symmetries on the Euclidean line $\mathbb{R}: \partial_{x}+\partial_{y}$ is a translation, $x \partial_{x}+y \partial_{y}$ is a dilation and $x^{2} \partial_{x}+y^{2} \partial_{y}$ is a conformal transformation [14. Therefore, the consideration of the conformal algebra on the $1 \mathrm{D}$ sphere $\mathbf{S}^{1}$ or hyperbolic line $\mathbf{H}^{1}$ may lead to the 'curved' analog of the $\mathrm{I}_{4}$-LH systems. We recall that $\mathrm{I}_{4}$ covers the so-called split-complex Riccati and coupled Riccati equations as well as some Milne-Pinney and Kummer-Schwarz equations (nondiffeomorphic to those of class $\left.\mathrm{P}_{2}\right)$ ].

- Finally, we also stress that the obtention of curved LH systems for the classes $\mathrm{P}_{2}$ and $\mathrm{I}_{4}$ could further provide a curved oscillator system with a time-dependent frequency and with a centrifugal or Winternitz term.

These problems are currently under investigation.

\section{Acknowledgments}

The research of F.J. Herranz was partially supported by the Spanish Ministerio de Economía y Competitividad (MINECO) under grant MTM2013-43820-P, by the grant MTM2016-79639-P (AEI/FEDER, UE) and by the Spanish Junta de Castilla y León under grants BU278U14 and VA057U16. J. de Lucas acknowledges funding from the Polish National Science Centre under grant MAESTRO (DEC2012/06/A/ST1/00256). M. Tobolski acknowledges partial support from the program Fizyka Plus with project number POKL.04.01.02-00-034/11-WF-37-18/13 carried out at the Faculty of Physics of the University of Warsaw and cofinanced by the European Union.

\section{References}

[1] Cariñena J F, Grabowski J and Marmo G 2000 Lie-Scheffers systems: a geometric approach (Naples: Bibliopolis)

[2] Cariñena J F and de Lucas J 2011 Lie systems: theory, generalisations, and applications, Dissertations Math. (Rozprawy Mat.) 479 1-162

[3] Lie S and Scheffers G 1893 Vorlesungen über continuierliche Gruppen mit geometrischen und anderen Anwendungen (Leipzig: Teubner)

[4] Winternitz P 1983 Nonlinear phenomena Lectures Notes in Physics 189 (New York: Springer) pp 263-331

[5] Cariñena J F, Grabowski J and Marmo G 2007 Rep. Math. Phys. 60 237-58

[6] Cariñena J F, Grabowski J and de Lucas J 2012 J. Phys. A: Math. Theor. 45185202

[7] Inselberg A 1972 J. Math. Anal. and Appl. 40 494-508

[8] Ballesteros A, Blasco A, Herranz F J, de Lucas J and Sardón C 2015 J. Differential Equations 258 2873-907

[9] Blasco A, Herranz F J, de Lucas J and Sardón C 2015 J. Phys. A: Math. Theor. 48345202

[10] Vaisman I 1994 Lectures on the geometry of Poisson manifolds (Basel: Birkhäuser)

[11] Marle C M 1997 J. Geom. Phys. 23 350-59

[12] Cariñena J F, de Lucas J and Sardón C 2013 Int. J. Geom. Methods Mod. Phys. 101350047 
[13] Ballesteros A, Cariñena J F, Herranz F J, de Lucas J and Sardón C 2013 J. Phys. A: Math. Theor. 46285203

[14] Grundland A M and de Lucas J 2017 J. Differential equations 263 299-337

[15] Anderson R L, Harnad J and Winternitz P 1981 Lett. Math. Phys. 5 143-148

[16] González-López A, Kamran N and Olver P J 1992 Proc. London Math. Soc. 64 339-68

[17] Shnider S and Winternitz P 1984 J. Math. Phys. 25 3155-3165

[18] Shnider S and Winternitz P 1984 Lett. Math. Phys. 8 69-78

[19] Brockett R 1973 SIAM J. Appl. Math. 25 213-25

[20] Komrakov B 1994 Proceedings Sophus Lie Memorial Conference Oslo 1992 ed Laudal O A and Jahren B (Oslo: Universitetsforlaget AS) pp 187-269

[21] Doubrov B M and Komrakov B P 1999 Geometry 83 Topology 3 1-20

[22] Cariñena J F, Clemente-Gallardo J, Jover-Galtier J A and de Lucas J 2016 preprint arXiv:1611.05630

[23] Yaglom I M 1979 A simple non-Euclidean geometry and its physical basis (New York: Springer)

[24] Rozenfel'd B A 1988 A history of non-euclidean geometry (New York: Springer)

[25] Gromov N A and Man'ko V I 1990 J. Math. Phys. 31 1047-53

[26] Gromov N A 1992 Contractions and analytical continuations of the classical groups. Unified approach (Syktyvkar: Komi Scienfic Center) (in Russian)

[27] Ballesteros A, Herranz F J, del Olmo M A and Santander M 1993 J. Phys. A: Math. Gen. 26 5801-23

[28] Herranz F J and Santander M 2002 J. Phys. A: Math. Gen. 35 6601-18

[29] Herranz F J, Ortega R and Santander M 2000 J. Phys. A: Math. Gen. 33 4525-51

[30] de Montigny M and Patera J 1991 J. Phys. A: Math. Gen. 24 525-47

[31] Moody R V and Patera J 1991 J. Phys. A: Math. Gen. 24 2227-57

[32] Herranz F J, de Montigny M, del Olmo M A and Santander M 1994 J. Phys. A: Math. Gen. 27 $2515-26$

[33] Campoamor-Stursberg R 2016 J. Math. Phys. 57063508

[34] Schuch D 2014 J. Phys.: Conf. Ser. 538012019

[35] Varadarajan V S 1984 Lie groups, Lie algebras, and their representations Graduate texts in Mathematics 108 (New York: Springer)

[36] Cariñena J F and López C 1999 Rep. Math. Phys. 43 43-51

[37] Patera J 1976 J. Math. Phys. 17 986-94

[38] Herranz F J and Santander M 1997 J. Phys. A: Math. Gen. 30 5411-26

[39] Ballesteros A, Blasco A, Herranz F J and Musso F 2014 J. Phys. A: Math. Theor. 47345204

[40] de Azcárraga J A, Herranz F J, Pérez Bueno J C and Santander M 1998 J. Phys. A Math. Gen. 31 1373-94 